\title{
Bildungsexpansion und un- gleiche Bildungspartizipation am Beispiel der Mittelschulen im Kanton Zürich, 1830 bis 1980
}

\section{Andreas Rieger}

Die Untersuchung der Mittelschulen im Kanton Zürich von 1830 bis 1980 zeigt deutlich, wie sich im Rahmen der Bildungsexpansions-Wellen die Bildungspartizipation verschiedener sozialer Gruppen wandelt: Frauen, Landbewohner und Katholiken können ihre Teilnahme an den Mittelschulen jener der Männer, Städter und Protestanten angleichen. Für die Kinder aus unteren sozialen Schichten - sowie der entsprechenden Nationalitäten - ist dies jedoch überhaupt nicht der Fall, sie bleiben die grosse benachteiligte Gruppe an den Zürcher Mittelschulen. Auf dem Hintergrund dieses Befundes stellt sich die Frage, warum die Diskussionen über die soziale Chancengleichheit trotz unerfüllten Ansprüchen versiegt sind.

\section{Einleitung, Fragestellung, Eingrenzungen}

Bildungsexpansion bedeutet, dass nicht allein die absoluten Schülerzahlen und die Schuldauer zunehmen, sondern dass die verschiedensten sozialen Schichten und Gruppen ihre Partizipation an der weiterführenden Bildung ausweiten können (Becker, 2000, S. 447 ff.). Zu Beginn von neuen Expansionswellen oder von Ausbauschritten im Bildungssystem wurde immer wieder argumentiert, dass damit bisher unterproportional partizipierende Gruppen vermehrte Chancen bekommen würden. Eine umfassende Bilanz, wie weit diese Versprechung eingelöst wurde, steht für die Schweiz bisher noch aus. Während die Aufholbewegung der Frauen im Bildungsbereich gut beschrieben wurde (u.a. Mantovani, 1994), ist dies für die Bildungspartizipation der unteren sozialen Schichten nicht der Fall. Die rege öffentliche Diskussion in den 60er-Jahren und anfangs der 70erJahren $^{1}$ verstummte mit wenigen Ausnahmen seit den 80 er-Jahren und die wissenschaftlichen Beiträge blieben einem kleineren Kreise vorbehalten (Bernath et al., 1989; Gurny et al., 1984; Haefeli et al., 1979; Karrer et al., 1991; Lamprecht \& Stamm, 1996, 1997).

Im vorliegenden Artikel wird versucht, eine Bilanz der Entwicklung der Bildungspartizipation verschiedener sozialer Gruppen zu skizzieren, die ursprün- 
glich deutlich unterproportional weiterführende Schulen besuchten oder gänzlich ausgeschlossen waren: Mädchen, Kinder vom Lande, Kinder aus der Unterschicht, Kinder von Immigranten sowie Katholiken.

Der Artikel legt dabei den Fokus auf den Besuch der Mittelschulen. Im Kanton Zürich werden als Mittelschulen die zur Maturität und freiem Hochschulzugang führenden Gymnasien sowie die mit einem kantonal anerkannten Diplom abschliessenden Vollzeit-Schulen der Sekundarstufe II bezeichnet. ${ }^{2}$ Aus Gründen des Umfangs wird die parallele Entwicklung im Bereich der beruflichen Ausbildung auf Sekundarstufe II ausgeblendet. ${ }^{3}$

Die Untersuchung wurde am Beispiel des Kantons Zürich durchgeführt, was eine Analyse einer ungebrochenen Entwicklung über 150 Jahre in einem institutionell einheitlichen Rahmen erlaubt. Dieser lange Betrachtungszeitraum lässt nicht nur die wellenförmig verlaufenden Prozesse der Bildungsexpansion deutlicher erkennen, sondern auch die Entwicklung der ungleichen Bildungspartizipation einzelner sozialer Gruppen. Die statistische Untersuchung reicht dabei bis in die 80er-Jahre des 20. Jahrhunderts.

\section{Vom demokratischen Neustart 1830 bis ins Jahr 1950}

Die liberal-demokratische Revolution im Kanton Zürich von 1830 brachte den definitiven Durchbruch des neuen öffentlichen Schulwesens, nachdem die Neuansätze der Schulpolitik der Helvetik in der nachfolgenden Restauration wieder weitgehend zurückgenommen worden waren. Auch die Mittelschulen und die Universität wurden in der Folge der 1830er-Revolution gegründet: Das kirchliche "Grossmünsterstift», welches bis dahin als einzige Institution eine höhere Ausbildung vermittelt hatte, wurde 1832 aufgelöst und seine Lehrer wurden entlassen. An die Stelle der von Kirche und Ancien Régime geprägten höheren Ausbildung traten staatliche und demokratisch kontrollierte Ausbildungsstätten: 1833 wurden als Mittelschulen die «Kantonsschule» (Gymnasium) und das «Lehrerseminar» gegründet. Im gleichen Jahr öffnete die «Universität» ihre Tore (Kronbichler, 1983). 1855, in der Folge der Gründung des Schweizerischen Bundesstaates von 1848, folgte die «Eidgenössische Technische Hochschule», ETH, in Zürich. Damit begann die erste Welle der «Bildungsexpansion» an den Mittelschulen.

\section{Erste Welle der Expansion des Mittelschulbesuchs}

Quantitativ gesehen war der Mittelschulbesuch anfangs noch sehr beschränkt: Für das Jahr 1850 können wir erstmals Mittelschüler-Quoten ${ }^{4}$ berechnen. Nur gerade 1,8\% eines Schülerjahrganges besuchten damals eine Mittelschule: 0,4\% das Lehrerseminar, 0,5\% das klassische Gymnasium, 0,9\% die «Industrieschule», wie damals die technisch ausgerichtete Mittelschule hiess. 
Die erste Expansionswelle kann für die Jahre 1850 bis 1890 festgestellt werden, insbesondere mit einem starken Wachstum der Diplommittelschulen «Handelsschule» und "Töchterschule». Besonders massiv war die Expansion sodann in der wirtschaftlichen Prosperitäts-Periode von 1890-1910: Die absolute Zahl der Mittelschülerinnen und Mittelschüler wuchs in diesen 20 Jahren von rund 1'000 auf 2'500 Schülerinnen und Schüler um rund 250\%, eine Wachstumsrate, die später nie mehr erreicht wurde (vgl. Tabelle 1).

Der Erste Weltkrieg und die nachfolgenden krisengeprägten Jahre bremsten dieses Wachstum bis Ende der 20er-Jahre ab. Danach aber wuchs trotz Weltwirtschaftskrise die Quote des Mittelschulbesuches wieder stark an, ermöglicht insbesondere durch konstant gehaltene Schulkapazitäten bei kleineren Geburtenjahrgängen.

Tabelle 1: Quoten des Mittelschulbesuchs am Gesamt eines Schülerjahrgangs, Kanton Zürich, 1850-19505

\begin{tabular}{|l|c|c|c|c|c|c|c|}
\hline Mittelschülerinnen und Mittelschüler & 1850 & 1870 & 1890 & 1910 & 1930 & 1941 & 1950 \\
\hline Literar-Gymnasium & 0,53 & 0,95 & 1,28 & 1,47 & 1,82 & 2,70 & 2,53 \\
\hline $\begin{array}{l}\text { Industrieschule/Mathematisch } \\
\text {-naturwissenschaftliches Gymnasium }\end{array}$ & 0,90 & 1,04 & 0,63 & 0,88 & 0,59 & 0,93 & 1,01 \\
\hline Handelsmaturitätsschule & - & - & - & 0,09 & 0,18 & 0,23 & 0,38 \\
\hline Handelsdiplomschule & - & 0,29 & 0,45 & 1,37 & 2,46 & 2,87 & 2,37 \\
\hline Lehrerinnen- und Lehrerseminar & 0,41 & 0,65 & 0,95 & 1,17 & 0,61 & 0,79 & 1,56 \\
\hline $\begin{array}{l}\text { «T̈̈chterschule» } \\
\text { Mittelschülerinnen und Mittelschüler total }\end{array}$ & - & - & 0,36 & 0,85 & 1,05 & 1,43 & 1,43 \\
\hline $\begin{array}{l}\text { davon Diplommittelschülerinnen } \\
\text { und Diplommittelschüler }\end{array}$ & 0,41 & 0,94 & 1,75 & 3,38 & 4,13 & 5,08 & 5,36 \\
\hline $\begin{array}{l}\text { davon Maturitätsmittelschülerinnen } \\
\text { und Maturitätsmittelschüler }\end{array}$ & 1,43 & 1,99 & 1,91 & 2,43 & 2,59 & 3,86 & 3,92 \\
\hline
\end{tabular}

Seit Anfang der 40er-Jahre des 20. Jahrhunderts wird das Wachstum deutlich gebremst. Dafür war nicht allein der Krieg verantwortlich; auch nach 1945 zog der Mittelschulbesuch noch lange nicht wieder an. Vielmehr ging bereits seit Mitte der 30er-Jahre mit der Weltwirtschaftskrise auch in der Schweiz das Gespenst der «akademischen Arbeitslosigkeit» um: 1935 konstatierte der Rektor der Universität Zürich bei den Universitäts-Absolventinnen und -absolventen eine Arbeitslosenrate von gegen 4,4 Prozent (Dünki, 1985, S. 19 f.). 1939 forderte die "Gesellschaft schweizerischer Akademiker» Zugangs-Restriktionen bei den Mittelschulen (Dünki, 1985, S. 22).

Diese alarmierenden Berichte hatten aber - abgesehen von den Lehrerseminaren $^{6}$ - keine Einführung eines Numerus Clausus zur Folge. Die Universitäten wollten sich nicht mit einem Numerus clausus und selektiven Prüfungen belasten; auch die Maturitätsschulen wehrten sich erfolgreich gegen die Relativierung des Maturaabschlusses und gegen einen Abbau der Anzahl Klassen. Aber auch 
ohne Numerus Clausus drückten Krise und Krieg auf die Quote der Mittelschülerinnen und Mittelschüler.

Am Ende des Krieges verschwand das Gespenst der «Akademikerarbeitslosigkeit». Es wirkte aber noch nach und hat dazu beigetragen, dass in den ersten $\mathrm{Na}$ chkriegsjahren keine neue Expansion des Mittelschulbesuches einsetzte. Noch 1951 plante der Zürcher Regierungsrat für die Mittelschulen nur einen Ausbau, welcher bei konstanter Mittelschülerquote das demographische Wachstum auffangen sollte. Ja noch 1958 prognostizierte das Rektorat der Universität Zürich eine Entwicklung der Studentenzahlen ohne jegliche Steigerung der Studentenquote (Rektorat Universität Zürich, 1983, S. 82).

Verglichen mit allen andern europäischen Ländern ist dies sehr untypisch, begannen die meisten von ihnen doch unmittelbar nach 1945 mit einer aktiven Nachwuchsförderung für die höhere Ausbildung und einem massiven Ausbau der entsprechenden schulischen Kapazitäten. Dass dies in der Schweiz nicht der Fall war, hat mit ihrer besonderen Situation am Ausgang des 2. Weltkriegs zu tun: Als einzige mit intaktem Produktionsapparat, setzte die Schweizer Wirtschaft auf ein einseitig extensives Wachstum unter voller Ausnutzung bestehender Kapazitäten. Die dennoch nötigen Spezialistinnen und Spezialisten (Ingenieure etc.) wurden problemlos auf dem europäischen Arbeitsmarkt rekrutiert. Weit verbreitet war zudem der - durch den Beginn des Kalten Kriegs geförderte - Glaube, der Nachkriegs-Boom sei nur von kurzer Dauer. Diese Einschätzung änderte sich erst Mitte der 50er-Jahre, nach dem Ende des Koreakriegs.

Die aussergewöhnliche Hemmung der Bildungsexpansion in der Schweiz hatte im europäischen Vergleich deutliche Folgen für die Studentenquoten: Befand sich die Schweiz 1920 mit einer Studierendenquote von rund 2\% bei den europäischen Vorreiter-Ländern (Frankreich und Niederlande 1,5\%, Italien 1,6\%, England und Deutschland 2\%), so war sie 1950 mit einer Studierendenquote von $3,7 \%$ bereits zurückgefallen und befand sich in der «Nachzüglergruppe» (Deutschland 4,4\%, Frankreich 4,3\%, Niederlande 7,6\%) (Kälble, 1983, S. 200).

\section{Die Entwicklung der Bildungschancen}

Die soziale Zusammensetzung der Mittelschülerinnen und Mittelschüler war in der beschriebenen ersten Phase der Expansion nur sehr begrenzt ein Thema der öffentlichen bildungspolitischen Diskussion. Dies erstaunt umso mehr, als der Neubeginn im liberal-demokratischen Zürich erwarten liesse, dass alle sozialen Gruppen der Gesellschaft einen Anspruch auf gleiche Chancen zum Besuch der Mittelschulen erhoben hätten.

\section{Soziale Chancen der Landbevölkerung}

Der Stadt-Land Gegensatz war einer der grossen gesellschaftlichen Widersprüche des 18. und beginnenden 19. Jahrhunderts im Kanton Zürich. Die Bildungsinstitutionen der Landschaft blieben im Ancien Régime und in der Re- 
staurationsperiode weit hinter denjenigen der Stadt zurück. Die Schaffung einer öffentlichen Volksschule, für welche in der Stadt wie in den Landgemeinden gleiche Regeln und gleiche Lehrpläne galten, gehörte zu den wichtigen Programmpunkten der liberalen Umwälzung von 1832, welche nur dank einer Allianz städtischer und ländlicher Klassen erfolgreich blieb.

Die Mittelschulen blieben dagegen auch nach 1832 auf die Städte Zürich und Winterthur begrenzt. Eine bezeichnende Ausnahme machte das Lehrerseminar, das in Küsnacht aufgebaut wurde, einem Hauptort des ländlichen Liberalismus. Es wurde bewusst mit einem Internat verbunden mit dem Ziel, Jugendliche vom Lande für die Lehrer-Ausbildung zu rekrutieren. Im Übrigen blieben die Kinder aus der Landbevölkerung, d.h. aus Gemeinden ausserhalb der Städte Zürich und Winterthur, lange eine klare Minderheit in den Mittelschulen, obwohl sie immer die Mehrheit in der Bevölkerung des Kantons stellten (vgl. Tabelle 2).

Tabelle 2: Anteil Mittelschülerinnen und Mittelschüler aus Landgemeinden, Kanton Zürich, 1900 bis $1950^{7}$

\begin{tabular}{|c|c|c|c|}
\hline Jahr & $\begin{array}{l}\text { Mittelschülerinnen und } \\
\text { Mittelschüler aus } \\
\text { Landgemeinden in \% aller } \\
\text { Mittelschülerinnen und } \\
\text { Mittelschüler im Kanton }\end{array}$ & $\begin{array}{l}\text { Einwohner in Land- } \\
\text { gemeinden in \% aller } \\
\text { Einwohner des Kantons }\end{array}$ & $\begin{array}{l}\text { 15-19-jährige Einwohner in } \\
\text { Landgemeinden in \% aller } \\
\text { Gleichaltrigen im Kanton }\end{array}$ \\
\hline 1900 & $24 \%$ & $52 \%$ & - \\
\hline 1930 & $31 \%$ & $44 \%$ & $53 \%$ \\
\hline 1950 & $34 \%$ & $41 \%$ & $53 \%$ \\
\hline
\end{tabular}

Diese Ungleichheit der Chancen der Landbevölkerung wurde erst in den 30erJahren des 20. Jahrhunderts als diskriminierend empfunden und führte zu politischen Vorstössen für eine eigene Mittelschule im Zürcher Oberland. 1946 gab der Regierungsrat ein entsprechendes Projekt in Auftrag, aber erst 1955 eröffnete die erste sogenannte «Landmittelschule» in Wetzikon ihre Tore.

\section{Frauen brechen Männerdomänen auf}

Waren die Mädchen im Konzept der demokratischen Volksschule auf Primarschulstufe von Anfang an mit einbezogen und in der Mehrheit der Sekundarschulen zumindest zugelassen, blieben sie von den Mittelschulen in den ersten Jahrzehnten völlig ausgeschlossen. Die in der Tabelle 1 "geschlechtsneutral» ausgewiesene Mittelschulquote von 1,8\% im Jahre 1850 war in Wirklichkeit eine Quote von 3,6\% der männlichen und von 0,0\% der weiblichen Jugendlichen (vgl. Tabelle 3). Erst in den 70er-Jahren des 19. Jahrhunderts wurde an der Töchterschule in der Stadt Zürich ein «Frauenfortbildungskurs» mit Diplomabschluss eröffnet und ebenso eine Lehrerinnen-Ausbildung. In den 90er-Jahren folgte die Handelsmittelschule. Seit 1904 war für Mädchen der Besuch eines Gymnasiums möglich (allerdings immer noch nur an der Töchterschule der 
Stadt Zürich und nicht an der «Kantonsschule», wo formell nur männliche Jugendliche zugelassen blieben). Durch dieses beharrliche Eindringen der Frauen in bisherige Männerdomänen wurde die massive Mittelschulexpansion von 1890-1910 zu einem grossen Teil verursacht. Dies gilt insbesondere im Bereich der Diplom-Mittelschulen, während die Mädchen im Gymnasium noch lange sehr schwach vertreten blieben.

Tabelle 3: Quoten des Mittelschulbesuchs nach Geschlecht, Kanton Zürich, 1850-1950

\begin{tabular}{|c|c|c|c|c|c|c|c|c|}
\hline & Geschlecht & 1850 & 1870 & 1890 & 1910 & 1930 & 1941 & 1950 \\
\hline \multirow[t]{2}{*}{ Literar-Gymnasium } & $\mathrm{F}$ & 0,00 & 0,00 & 0,00 & 0,52 & 1,05 & 1,56 & 1,54 \\
\hline & $\mathrm{M}$ & 1,06 & 1,89 & 2,54 & 2,36 & 2,59 & 3,85 & 3,52 \\
\hline \multirow{2}{*}{$\begin{array}{l}\text { Mathematisch-naturwissen- } \\
\text { schaftliches Gymnasium }\end{array}$} & $\mathrm{F}$ & 0,00 & 0,00 & 0,00 & 0,02 & 0,02 & 0,01 & 0,01 \\
\hline & $\mathrm{M}$ & 1,80 & 2,06 & 1,26 & 1,68 & 1,15 & 1,85 & 2,01 \\
\hline \multirow[t]{2}{*}{ Handelsmaturitätsschule } & $\mathrm{F}$ & - & - & - & 0,00 & 0,00 & 0,08 & 0,26 \\
\hline & M & - & - & - & 0,16 & 0,36 & 0,39 & 0,49 \\
\hline \multirow[t]{2}{*}{ Handelsdiplomschule } & $\mathrm{F}$ & - & 0,00 & 0,00 & 0,85 & 2,35 & 2,41 & 2,33 \\
\hline & $\mathrm{M}$ & - & 0,58 & 0,89 & 1,86 & 2,58 & 3,32 & 2,42 \\
\hline \multirow{2}{*}{$\begin{array}{l}\text { Lehrerinnen- und } \\
\text { Lehrerseminar }\end{array}$} & $\mathrm{F}$ & 0,00 & 0,00 & 0,70 & 1,21 & 0,51 & 0,58 & 1,80 \\
\hline & M & 0,81 & 1,30 & 1,18 & 1,13 & 0,72 & 0,99 & 1,31 \\
\hline \multirow[t]{2}{*}{ «Töchterschule» } & $\mathrm{F}$ & - & - & 0,74 & 1,74 & 2,11 & 2,86 & 2,87 \\
\hline & $\mathrm{M}$ & - & - & 0,00 & 0,00 & 0,00 & 0,00 & 0,00 \\
\hline \multirow{2}{*}{$\begin{array}{l}\text { Mittelschülerinnen und } \\
\text { Mittelschüler total }\end{array}$} & $\mathrm{F}$ & 0,00 & 0,00 & 1,42 & 4,35 & 6,04 & 7,49 & 8,82 \\
\hline & $\mathrm{M}$ & 3,67 & 5,82 & 5,87 & 7,19 & 7,40 & 10,39 & 9,75 \\
\hline \multirow{2}{*}{$\begin{array}{l}\text { davon Diplommittel- } \\
\text { schülerinnen und -schüler }\end{array}$} & $\mathrm{F}$ & 0,00 & 0,00 & 1,42 & 3,80 & 4,96 & 5,58 & 7,00 \\
\hline & $\mathrm{M}$ & 0,81 & 1,88 & 2,07 & 2,99 & 3,30 & 4,31 & 3,73 \\
\hline \multirow{2}{*}{$\begin{array}{l}\text { davon Maturitätschüler- } \\
\text { schülerinnen und -schüler }\end{array}$} & $\mathrm{F}$ & 0,00 & 0,00 & 0,00 & 0,54 & 1,08 & 1,64 & 1,81 \\
\hline & $\mathrm{M}$ & 2,86 & 3,94 & 3,80 & 4,20 & 4,10 & 6,08 & 6,02 \\
\hline
\end{tabular}

In der Zwischenkriegszeit stieg der Mittelschulbesuch der weiblichen Jugendlichen kontinuierlich an. Sie und ihre Eltern liessen sich offensichtlich von Krisen und Klagen über Akademikerarbeitslosigkeit weniger einschüchtern ${ }^{8}$ als dies bei der männlichen Jugend der Fall war, für welche die pessimistische wirtschaftliche Aussicht offensichtlich einflussreicher war: Die Mittelschülerquote für männliche Jugendliche verläuft von 1941 bis 1950 sogar rückläufig!

Nach vielen Kämpfen, individuellen wie auch politischen, hatten die weiblichen Jugendlichen 1950 mit einer Mittelschülerquote von 8,8\% schon beinahe jene der männlichen $(9,7 \%)$ erreicht; allerdings war dies immer noch wesentlich auf ihre Übervertretung in den Diplommittelschulen zurückzuführen (vgl. Tabelle 3). 


\section{Extreme Ungleichheit zwischen sozialen Schichten}

Die ungleichen Chancen verschiedener sozialer Schichten hinsichtlich des Mittelschulbesuchs waren in dieser langen Periode kein Thema der Auseinandersetzung. Kaum jemand stellte in Frage, dass Kinder aus Arbeiter- und Bauernfamilien - damals immer noch die Mehrheit der Gesellschaft - an den Maturitätsschulen und Universitäten quasi abwesend waren. Für die Arbeiterbewegung standen schulpolitisch damals andere Fragen im Vordergrund: der Kampf gegen die Spaltung der Sekundarstufe I in eine "Armenschule» (7./8. Primarklassen) und eine qualitativ gute «Sekundarschule» sowie der Kampf für gesetzlich geregelte gute Arbeits- und Lernbedingungen der Lehrlinge und unentgeltlichen Zugang zu den Berufsschulen. Bis in die 50er-Jahre des 20. Jahrhunderts bestand ein «entscheidender Zug aller bisherigen Bildungspolitik

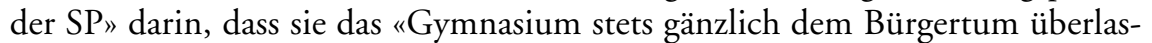
sen hatte» (Nabholz, 1981).

Bis in die 50er-Jahre wirkten einige bewusst gesetzte Barrieren, wie die Bezahlung von Prüfungs-, Eintritts- und Schulgeldern, sowie der Lehrmittel (Schmid-Ammann, 1957, S. 3 f.). Noch 1930 verlangte das Stipendienregulativ für einen Studienbeitrag nicht nur den Nachweis ökonomischer Bedürftigkeit, sondern auch besondere "Begabung, Leistung, Fleiss und Wohlverhalten» (zitiert nach Schmid-Ammann, 1957, S. 4). Diese materiellen Barrieren kombinierten sich mit einer offen elitären Abwehrhaltung seitens der Bildungsverantwortlichen, wie sie der erste «akademische Studienberater» von Zürich deutlich ausdrückte:

"Eine grosse Zahl von Leuten, die früher eine Berufslehre als selbstverständlich angesehen hätten, verschmähen diese heute und drängen sich an die Mittelschulen [...]. Ihr Studienmotiv ist nicht eigentlich das Studieren und der akademische Beruf, sondern sie sehen darin das Mittel zum sozialen Aufstieg. Sie sind es dann aber auch, die später [...] einfach versagen müssen und das akademische Proletariat abgeben. Es wird also vieles davon abhängen, ob es gelingt, diese Bewegung in eine rückläufige zu verwandeln» (Ungricht, 1944, S. 7).

Eine Ausnahme machte das Lehrerseminar, das schulbegabte Jugendliche aus Arbeiter- und Bauernkreisen von Anfang an mit Erfolg förderte: Bereits 1838 kamen von 45 Schülern 21 aus Bauern-, 12 aus Handwerkerfamilien, 24 brauchten ein Stipendium (Schmid, 1982, S. 23).

Erste - in Tabelle 4 zusammengestellte - Statistiken zeigen für die Hochschulstudentinnen und -studenten ein relativ einheitliches Bild des schichtspezifischen Zugangs zur höheren Ausbildung: 
Tabelle 4: Studierende nach sozialer Herkunft in Prozent aller Studierenden, 1936 bis $1959^{9}$

\begin{tabular}{|c|c|c|c|c|c|}
\hline $\begin{array}{l}\text { Beruf/Stellung } \\
\text { des Vaters }\end{array}$ & Hochschule & $\begin{array}{c}1935 \\
\text { (Schweiz) }\end{array}$ & $\begin{array}{c}1945 \\
\text { (Schweiz) }\end{array}$ & $\begin{array}{l}1945-50 \\
\text { (Zürich) }\end{array}$ & $\begin{array}{c}1959 \\
\text { (Schweiz) }\end{array}$ \\
\hline $\begin{array}{l}\text { Liberale Berufe } \\
\text { (Anwälte, Lehrer, } \\
\text { Ingenieure, Pfarrer usw.) }\end{array}$ & $\begin{array}{l}\text { ETH } \\
\text { Uni, Männer } \\
\text { Uni, Frauen } \\
\end{array}$ & $\begin{array}{l}40^{*} \\
35 \\
45 \\
\end{array}$ & & \} ca. 32 & $\begin{array}{l}25 \\
30 \\
36 \\
\end{array}$ \\
\hline Arbeiter & $\begin{array}{l}\text { ETH } \\
\text { Uni, Männer } \\
\text { Uni, Frauen } \\
\end{array}$ & $\begin{array}{c}3,8^{*} \\
6,2 \\
3,3 \\
\end{array}$ & $\begin{array}{l}3,6 \\
5,8 \\
2,3 \\
\end{array}$ & 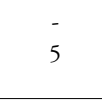 & $\begin{array}{c}4,1 \\
6 \\
2 \\
\end{array}$ \\
\hline Angestellte und Beamte & $\begin{array}{l}\text { ETH } \\
\text { Uni, Männer } \\
\text { Uni, Frauen }\end{array}$ & $\begin{array}{c}27^{*} \\
31 \\
26\end{array}$ & & - 35 & $\begin{array}{l}46 \\
43 \\
42\end{array}$ \\
\hline $\begin{array}{l}\text { Selbständige } \\
\text { (ohne Liberale Berufe) }\end{array}$ & $\begin{array}{l}\text { ETH } \\
\text { Uni, Männer } \\
\text { Uni, Frauen }\end{array}$ & $\begin{array}{c}27^{*} \\
26 \\
23 \\
\end{array}$ & & - 28 & $\begin{array}{l}25 \\
20 \\
19 \\
\end{array}$ \\
\hline
\end{tabular}

*Schweizer und Ausländer

Kinder von Arbeitern - damals rund 50\% der Erwerbstätigen - stellen in allen diesen Untersuchungen also nur durchschnittlich 5 Prozent der Studierenden. Kinder von Angestellten und Beamten sind dagegen an den Hochschulen mit 30-40\% etwas besser vertreten als es ihrem Anteil an den männlichen Erwerbstätigen - damals rund 20\% der Erwerbstätigen - entsprach. Kinder von Vätern mit «liberalen» Berufen (1930 rund 7\% der männlichen Erwerbstätigen) sind im Verhältnis zu ihrem Anteil an den Erwerbstätigen am stärksten präsent.

Welches die Wahrscheinlichkeit des Hochschulbesuchs für Kinder von Vätern ausgewählter Berufsgruppen ist, zeigen die Studentenquoten, welche sich für ausgewählte Berufsgruppen errechnen lassen. Sie drücken erst richtig das riesige Ausmass der Ungleichheit der Partizipation an höherer Ausbildung aus:

Tabelle 5: Quoten des Hochschulbesuchs nach ausgewählten Berufen der Väter, Schweiz, 193510

\begin{tabular}{|ll|lc|}
\hline Schreiner & 0,6 & Buchhalter & 4 \\
\hline Mechaniker & 0,6 & Techniker & 10,2 \\
\hline Magaziner & 0,3 & Postbeamter & 9,4 \\
\hline Briefträger & 0,9 & Kaufmänn. Ang. & 8,1 \\
\hline Maurer & 0,2 & Volksschullehrer & 12,4 \\
\hline Zugführer/Kondukteure & 2 & Ärzte & 30,7 \\
\hline Metzger & 1,6 & Hochschullehrer & 67 \\
\hline Schneider & 1 & Mittelschullehrer & 35 \\
\hline Schuhmacher & 0,5 & Ingenieure & 40 \\
\hline
\end{tabular}


Bei einer generellen durchschnittlichen Wahrscheinlichkeit des Hochschulbesuchs von 3\% lag die Wahrscheinlichkeit bei Kindern von Arbeitern und Gewerblern zwischen 0,2 und 1,6 Prozent; jene der Kinder von Ärzten, Hoch- und Mittelschullehrern dagegen bei 30 bis über $60 \%$ ! Zu einem grossen Teil rekrutierten sich die Mittelschülerinnen und Mittelschüler bzw. die Studentinnen und Studenten aus den Kreisen des Bildungsbürgertums, der höheren Beamten und der Angestellten. In Unternehmerschichten dagegen war ein Hochschulstudium zwar eine Möglichkeit, jedoch keineswegs besonders häufig angestrebt. Unternehmer planten für ihre Nachkommen oft eine individuelle, massgeschneiderte Ausbildung, welche die Mitarbeit im eigenen Unternehmen (z.T. auch in der Produktion, "von der Pike auf») mit Praktika in andern Betrieben, Auslandaufenthalten und dem Besuch von Schulen kombinierte (vgl. dazu auch Bourdieu \& Boltanski, 1981).

\section{Die abwesende katholische Minderheit}

Der Kanton Zürich war als Wiege der zwinglianischen Reformation lange völlig protestantisch dominiert. Seit Ende des 19. Jahrhunderts strömten jedoch zunehmend katholische Immigrantinnen und Immigranten aus Deutschland, Italien sowie aus der Inner- und Ostschweiz nach Zürich und sahen sich hier als religiös-kulturelle Minderheit diskriminiert. Die bewussten Katholiken organisierten sich als sogenannte "Diaspora» in einem Gegenmilieu, das - ähnlich dem Milieu der sozialdemokratischen Arbeiterschaft - das Leben «von der Wiege bis zur Bahre» zu organisieren versuchte (Altermatt, 1989, S. 181 ff.). Im Unterschied zur Arbeiterbewegung waren die Katholiken aber nicht an der öffentlichen Schule orientiert, sondern bauten als eigene Institution die "Katholische Sekundarschule» auf. Daran anschliessend kam für bewusste katholische Eltern ein Besuch der offen antiklerikal eingestellten öffentlichen Gymnasien - an welchen bis Anfang der 1960er-Jahre kaum ein katholischer Lehrer angestellt wurde - nicht in Frage ${ }^{11}$. Katholische Zürcher Jugendliche, welche eine Mittelschule besuchen wollten, wichen deshalb in die Knabeninternate in Einsiedeln, Schwyz, Engelberg oder Altdorf, respektive nach Menzingen, Ingenbohl und andere Mädcheninternate aus.

Leider ist dieser Prozess statistisch nicht rekonstruierbar: Die Erziehungsbehörden nahmen das Problem nicht wahr, sodass die Konfessionszugehörigkeit der Mittelschülerinnen und Mittelschüler gar nicht erfasst bzw. nicht publiziert wurde. Umgekehrt ist die Kantonsherkunft der Schülerinnen und Schüler der Internate für diese weit zurückliegenden Jahrzehnte nicht mehr zu eruieren. Das institutionelle Muster des Mittelschulbesuchs war bei den Katholiken jedenfalls grundlegend anders als bei den Zürcher Protestanten.

Zwischenstand am Ende der ersten Expansionswelle Bis Mitte der 1950er-Jahre waren die sozialen Chancen für einen Mittelschulbesuch - und mehr noch für das Erlangen der Maturität - extrem ungleich verteilt. 
Die von der ersten Welle der Bildungsexpansion im Kanton Zürich profitierenden Mittelschülerinnen und Mittelschüler erweisen sich bei genauerem Hinsehen grossmehrheitlich als aus der städtischen, protestantischen Mittel- und Oberschicht stammend. Oder wie ein Gymnasiallehrer 1959 treffend bemerkte: Das Gymnasialsystem blieb «hauptstädtisch und plutokratisch, wie wenn sich die kantonalen Revolutionen von 1830 nie ereignet hätten» (Im Hof, 1959, S. 27 f. ). Die Chancen eines katholischen Arbeiter- oder Bauern-Mädchens vom Lande, ein Zürcher Gymnasium zu besuchen, waren hundertemal kleiner als jene eines protestantischen Sohnes eines Hochschullehrers oder Arztes in der Stadt.

Dass diese Tatsache bis in die 50er-Jahre des 20. Jahrhunderts nur so wenig politische Auseinandersetzungen ausgelöst hat, mag heute erstaunen. Es ist nur erklärlich, wenn wir den begrenzten gesellschaftlichen und machtpolitischen Stellenwert der höheren Ausbildung im Auge behalten: Bis in die 50er-Jahre war die Universität nur wichtig für den Zugang zu einigen spezialisierten Berufen (Arzt, Jurist, Pfarrer, Ingenieur) sowie zu den Lehrberufen. Die grosse Mehrheit der gesellschaftlich mächtigen Berufstätigkeiten war an keinerlei höhere Ausbildung gebunden. Dies galt sowohl für die private Wirtschaft, deren Spitzenkader mehrheitlich eine Berufslehre oder allenfalls eine Handelsschule absolviert hatten, aber auch für den Staat, der auch für Spitzenbeamten-Posten, ja nicht einmal für das Richteramt ${ }^{12}$, eine höhere Ausbildung voraussetzte. Entsprechend war der Zugang zur höheren Ausbildung noch keineswegs notwendige Bedingung für einen sozialen Aufstieg. Schulpolitisch umkämpft waren in dieser Periode deshalb vor allem die Zugänge zur Sekundarschule sowie zu den qualitativ hochstehenden Berufslehren und Berufsschulen.

\section{Die Expansion der Mittelschulen ab Mitte der 1950er-Jahre}

\section{Der Anstoss zur zweiten Expansionswelle}

Der Anstoss zur neuen Expansionswelle der Mittel- und Hochschulen kam im Kanton Zürich - wie in der Mehrheit der Schweizer Kantone - erst seit Mitte der 50er-Jahre des 20. Jahrhunderts, und zwar von Seiten der Wirtschaft. 1955 zog der Arbeitgeberverband der Schweizer Maschinenindustrie nach einer Bedarfsumfrage die Alarmglocke (Aebli, 1963, S. 14): Für die Zukunft sei mit einem grossen Defizit an Ingenieuren zu rechnen. Im gleichen Jahr konstatierte Fritz Hummler, Delegierter des Bundesrats für «Arbeitsbeschaffung und Kriegsvorsorge», für weite Bereiche von Industrie und Forschung ein Arbeitskräftedefizit und warnte, die Schweiz drohe im internationalen Wettbewerb ins Hintertreffen zu geraten. Im Jahr darauf versammelte er alle Wirtschaftsverbände und viele «Industriekapitäne», welche die Besorgnis über ein Qualifikationsdefizit zum Ausdruck brachten und einen Arbeitsausschuss ins Leben riefen (Arbeits- 
ausschuss, 1959, S. 7, 52). Dieser führte 1957 auf der Arbeitgeberseite eine Umfrage zur Abklärung des Bedarfs an Ingenieuren, Technikern und Naturwissenschaftern durch, welche für die kommenden 10 Jahre eine riesige Nachwuchslücke ergab. Daraus wurden klare Forderungen abgeleitet, unter anderem der massive Ausbau der Mittelschulen, die Gleichstellung des Gymnasialtypus C mit den andern Typen sowie eine Erhöhung der Kapazitäten der Hochschulen und der Technika (Arbeitsausschuss, 1959, S. 15 ff.).

Später wurde auch der Bedarf in den andern akademischen Disziplinen errechnet, vor allem bei den Mittel- und HochschullehrerInnen, aber auch bei den JuristInnen und MedizinerInnen, und auch hier ein deutlicher Mangel festgestellt (Eidgenössische Kommission für Nachwuchsfragen, 1963).

Diese Bedarfsanalysen wurden in der Folge durch die rasante Wirtschaftsentwicklung anfangs der 60er-Jahre und die immer stärkere Anspannung des Arbeitsmarkts in der Praxis bestätigt. Die Ende der 50er-Jahre gestellten Forderungen nach zusätzlichen hochqualifizierten Arbeitskräften erwiesen sich sogar als zu kurz gegriffen. Dies traf auch auf den Kanton Zürich zu, dessen florierende Industrie besonders auf zusätzliches akademisches Personal angewiesen war und deshalb massiv auf Ausländerinnen und Ausländer zurückgriff. Diese stellten gemäss Volkszählung 1960 im Kanton Zürich bereits 27 Prozent der berufstätigen Maschinen-Ingenieure, 24 Prozent der Chemiker und 27 Prozent der Mathematiker. In einigen Dokumenten wurde in der Folge bereits die Gefahr der «Überfremdung» durch ausländische Akademiker aufgeführt (Arbeitsausschuss, 1959, S. 3, 8).

1957 schickte die Sowjetunion den ersten Sputnik ins Weltall und setzte damit im Wettbewerb zwischen den Blöcken ein Zeichen, das im öffentlichen Diskurs des kalten Krieges eine wichtige Rolle spielte, auch bezüglich der Bildungspolitik: «Deshalb kann Nachwuchsplanung [...] als unentbehrliches Mittel zur Stärkung einer freien Gesellschaft betrachtet werden», war im Bericht der «Eidgenössischen Kommission» zu lesen (Eidgenössische Kommission für Nachwuchsfragen, 1963, S. 211). Im Organ der Mittelschullehrer «Gymnasium Helveticum» warf Ulrich Im Hof den Gegnern einer Öffnung der Gymnasien gar «Sabotage an der wirtschaftlichen Verteidigung» vor (Im Hof, 1959, S. 29).

Schliesslich wurde in der öffentlichen Diskussion auch konstatiert, dass in unteren sozialen Schichten grössere «Begabungsreserven brachliegen». Dies und nicht etwa demokratie- oder sozialpolitische Überlegungen - gaben den Anstoss zur Zürcher Diskussion über vermehrte Chancengleichheit im Bildungswesen.

1964 publizierte der Zürcher Erziehungsrat das Dokument «Aktuelle Mittelschulfragen» (Erziehungsrat des Kantons Zürich, 1964), in welchem er all diese Argumente für einen Ausbau der Mittelschulen zusammenfasste und den Aufbau einer eigentlichen Bildungsplanung forderte, welche namentlich folgende Reformen anleiten sollte: 
- Gründung neuer Mittelschulen in den Land-Regionen,

- Abschaffung der Schulgelder und Verbesserung der Stipendien,

- Aufwertung und Ausbau der Kurzzeitgymnasien,

- Schaffung eines neuen, sprachlich orientierten Matura-Typus D und

- Aufwertung der Diplommittelschulen.

Dieses Programm haben Zürcher Regierung und Erziehungsbehörden erfolgreich in rund einem Dutzend Volksabstimmungen, die für gesetzliche Änderungen und Baukredite nötig waren, vertreten und durchgesetzt. Getragen wurde diese Politik von einer Allianz des Landesrings der Unabhängigen (diese damals starke «Angestellten»-Partei stellte lange den Erziehungsdirektor), der Sozialdemokratischen Partei und der Freisinnig-Liberalen Partei. Meist erfolglos versuchte die Bauern-, Gewerbe- und Bürgerpartei, die heutige SVP (Schweizerische Volkspartei), den Mittelschulausbau zu bremsen.

Mittelschul- und Hochschulpolitik war in dieser Phase ein "FortschrittsThema», mit dem sich Politiker Verdienste erwerben konnten. "Seit 1958 hatte die Stimmung umgeschlagen. Sowohl im Kanton Zürich wie auch in der Eidgenossenschaft waren 1959/61 die Universitätsprobleme nicht nur zu einem Politikum ersten Ranges, sondern zu einem eigentlichen Lieblingskind der Politiker und der ganzen Öffentlichkeit geworden» (Rektorat Universität Zürich \& Stadler, 1983, S. 99). Je nach Klientel verwendeten die Politiker dabei unterschiedliche Begründungen für den Mittelschulausbau: Zur Überzeugung von Arbeitern wurde natürlich das Argument der vernachlässigten Begabungen von Arbeiterkindern bevorzugt, zur Mobilisierung der "Landschaft» der Abbau der Barrieren ausserhalb der grossen Städte; für konservatives Publikum waren dagegen die System-Wettbewerbsargumente angebrachter, aber auch das Argument der drohenden "Überfremdung» durch ausländische Akademiker. Bis in die 70er-Jahre hinein gewann die «Bildungsallianz» damit beinahe alle Volksabstimmungen.

\section{Das Ausmass der neuen Expansion}

Auf diesem Hintergrund kam seit Ende der 50er-Jahre die zweite Welle der Expansion höherer Bildung ins Rollen. Der Andrang der Schülerinnen und Schüler in die neu gegründeten Schulen und Schultypen blieb nicht aus. Ja die Entwicklung der Schülerzahlen an den Mittelschulen ging weit über die Expansionspläne der Bildungspolitiker hinaus. Von 1960 bis 1980 verdoppelte sich die absolute Zahl der Mittelschülerinnen und Mittelschüler von 7’000 auf 14 ’000 (vgl. Tabelle 6). 
Tabelle 6: Quoten des Mittelschulbesuchs am Gesamt eines Schülerjahrgangs, Kanton Zürich, 1941-1980

\begin{tabular}{|l|c|c|c|c|c|}
\hline & 1941 & 1950 & 1960 & 1970 & 1980 \\
\hline Literar-Gymnasium & 2,70 & 2,53 & 3,08 & 3,99 & 4,69 \\
\hline $\begin{array}{l}\text { Mathematisch-naturwissen- } \\
\text { schaftliches Gymnasium }\end{array}$ & 0,93 & 1,01 & 1,46 & 1,86 & 2,47 \\
\hline Handelsmaturitätsschule & 0,23 & 0,38 & 0,30 & 1,13 & 1,55 \\
\hline Handelsdiplomschule & 2,87 & 2,37 & 1,90 & 1,71 & 1,32 \\
\hline Lehrerinnen- und Lehrerseminar & 0,79 & 1,56 & 2,21 & 2,28 & 2,51 \\
\hline «Töchterschule» resp. DMS* & 1,43 & 1,43 & 1,61 & 1,78 & 1,55 \\
\hline Neusprachliches Gymnasium & & & & 0,28 & 1,02 \\
\hline $\begin{array}{l}\text { Mittelschülerinnen } \\
\text { und Mittelschüler total }\end{array}$ & 8,94 & 9,28 & 10,56 & 12,75 & 15,10 \\
\hline $\begin{array}{l}\text { davon Diplommittelschülerinnen } \\
\text { und Diplommittelschüler }\end{array}$ & 5,08 & 5,36 & 5,72 & 5,77 & 5,38 \\
\hline $\begin{array}{l}\text { davon Maturitätsmittelschülerinnen } \\
\text { und Maturitätsmittelschüler }\end{array}$ & 3,86 & 3,92 & 4,84 & 6,98 & 9,72 \\
\hline
\end{tabular}

* In den 70er-Jahren wurde - in Fortsetzung der «Töchterschule» - die auch für Knaben geöffnete «Diplommittelschule» (DMS) geschaffen.

Besonders stark war dabei die Zunahme bei den Maturitätsschülerinnen und -schülern, während die erhoffte Aufwertung der mit einem Diplom abschliessenden Mittelschulen ausblieb. Der Drang zur Maturität drückte sich schliesslich auch bei der Lehrerinnen- und Lehrerbildung aus, deren Abschluss in dieser Zeit in Richtung allgemeinem Hochschulzugang aufgewertet wurde.

Bereits Mitte der 70er-Jahre war jedoch die Zeit vorbei, in der die Mittel- und Hochschulpolitik «Lieblingskind» der Politiker und des Stimmvolks war. Bereits 1973 bei der Volksabstimmung um den neuen Bildungsartikel in der Bundesverfassung zeichnete sich die Trendumkehr ab: Gegen das vage formulierte «Recht auf Bildung» sowie gegen die verstärkte Bundeskompetenz scharte sich eine grosse bürgerliche Opposition, welche in der Volksabstimmung obsiegte. In der Rezession 1974-76 tauchte sodann wieder das «Gespenst» der «AkademikerArbeitslosigkeit» auf und war für die scharf auf die Ausgabenbremse tretende öffentliche Hand, auch für den Kanton Zürich, Anlass zu Sparprogrammen im Bildungsbereich. Der öffentliche Diskurs über höhere Bildung änderte sich drastisch, mit der Forderung nach ihrem Ausbau wollte sich kein Politiker mehr profilieren.

Diese Änderung der politischen Konjunktur hat die Expansion des Mittelschulbesuchs zwar etwas abgebremst, aber nicht gestoppt. Indem kleinere Geburtenjahrgänge ins Mittelschulalter gelangten, war auch ohne Mittelschulausbau ein weiteres Ansteigen der Mittelschülerquoten bis auf über 20 Prozent in den 90er-Jahren möglich. 
Entwicklung der sozialen Chancen im neuen

Expansionsschub

Wie hat sich nun die Partizipation der verschiedenen sozialen Gruppen im Rahmen der zweiten Expansionswelle entwickelt?

\section{Aufhebung des Stadt-Land-Gefälles}

Das Expansionsprogramm seit Mitte der 50er-Jahre beinhaltete als ein wesentliches Element die «Dezentralisierung der Mittelschulen», d.h. die Eröffnung von Mittelschulen in ländlichen Zentren (Wetzikon, Bülach, Urdorf) und zusätzlich die Ausrichtung einzelner städtischer Mittelschulen auf ihr benachbartes Agglomerationsumfeld. Dieses Programm wurde mit Erfolg umgesetzt:

Tabelle 7: Anteil Mittelschülerinnen und Mittelschüler aus Landgemeinden, Kanton Zürich, 1950 und 1980

\begin{tabular}{|l|c|c|c|}
\hline & $\begin{array}{l}\text { Mittelschülerinnen und Mittel- } \\
\text { schüler aus Landgemeinden in } \\
\% \text { aller Mittelschülerinnen } \\
\text { und Mittelschüler im Kanton }\end{array}$ & $\begin{array}{l}\text { Einwohner in Land- } \\
\text { gemeinden in \% aller } \\
\text { Einwohner des Kantons }\end{array}$ & $\begin{array}{l}\text { 15-19-jährige Einwohner in } \\
\text { Landgemeinden in \% aller } \\
\text { Gleichaltrigen im Kanton }\end{array}$ \\
\hline 1950 & $34 \%$ & $41 \%$ & $53 \%$ \\
\hline 1980 & $65 \%$ & $45 \%$ & $65 \%$ \\
\hline
\end{tabular}

Seit 1980 sind die Landbezirke als Ganzes gegenüber der Stadt nicht mehr benachteiligt. Geblieben ist zwar ein sehr unterdurchschnittlicher Mittelschulbesuch in vielen Gemeinden ausserhalb der Städte Zürich und Winterthur; dies wird aber kontrastiert durch einen sehr überdurchschnittlichen Mittelschulbesuch in anderen Gemeinden ausserhalb der Städte. Diese Tatsache ist nun aber nicht mehr auf einen Stadt-Land-Unterschied zurückzuführen, sondern klar auf die soziale Zusammensetzung der Gemeinden (Frei, 1986).

Diese Auflösung des Stadt-Land-Unterschieds beim Mittelschulbesuch ist natürlich nicht allein der institutionellen Dezentralisierung zu verdanken. Hintergrund sind die strukturellen Veränderungen des sozialen Raumes mit der Herausbildung der "Grossagglomeration Zürich» (Kägi, 1991).

\section{Frauen: Hauptreservoir der neuen Bildungsexpansion}

Bereits 1950 hatten die Mädchen beim Mittelschulbesuch beinahe die Quote der Knaben erreicht, allerdings noch vor allem dank besonders hohen Quoten bei den Diplommittelschulen.

Bei der Diskussion über den Ausbau der Mittelschulen in den 50er-Jahren spielte nun interessanterweise die Frage der vermehrten Förderung von Frauen kaum eine Rolle. In der Aufzählung der ausschöpfbaren «Begabungs-Reserven» war zwar immer die Rede von den Jugendlichen auf dem Lande sowie von den Kindern von Arbeitern, Bauern und unteren Angestellten, selten jedoch von den Frauen. Die mögliche Angleichung der Gymnasialquoten von Frauen und Män- 
nern spielte in den Zielvorgaben des Mittelschulausbaus keinerlei Rolle. ${ }^{13}$ Ganz überraschend oder zufällig war dieses "Vergessen» der Frauen aber keineswegs. Die Argumentation für den Mittelschulausbau ging sehr stark vom Bedarf der Industrie und Wissenschaft nach zusätzlichen hochqualifizierten Arbeitskräften aus. Wenn davon die Rede war, man brauche immer mehr Ingenieure, Techniker, Naturwissenschafter usw., so waren damit effektiv Männer gemeint. ${ }^{14}$ In der Realität waren dann jedoch die Mädchen das Hauptreservoir, aus dem die Mittelschul-Expansion von 1950 bis 1980 schöpfte. Sie drängten nun vor allem auch in die Mittelschultypen mit Hochschulzugang und verfünffachten hier ihre Quote, während die in der Planung der Expansion hauptsächlich anvisierten Knaben ihre Quote kaum verdoppelten (vgl. Tabelle 8).

Tabelle 8: Quoten des Mittelschulbesuchs nach Geschlecht, Kanton Zürich, 1950-1980

\begin{tabular}{|c|c|c|c|c|c|}
\hline & Geschlecht & 1950 & 1960 & 1970 & 1980 \\
\hline \multirow[t]{2}{*}{ Literar-Gymnasium } & $\mathrm{F}$ & 1,54 & 2,24 & 3,21 & 4,78 \\
\hline & M & 3,52 & 3,87 & 4,73 & 4,60 \\
\hline \multirow{2}{*}{$\begin{array}{l}\text { Mathematisch-naturwissen- } \\
\text { schaftliches Gymnasium }\end{array}$} & $\mathrm{F}$ & 0,01 & 0,02 & 0,39 & 0,80 \\
\hline & M & 2,01 & 2,81 & 3,24 & 4,06 \\
\hline \multirow[t]{2}{*}{ Handelsmaturitätsschule } & $\mathrm{F}$ & 0,26 & 0,14 & 0,69 & 1,32 \\
\hline & M & 0,49 & 0,45 & 1,55 & 1,75 \\
\hline \multirow[t]{2}{*}{ Handelsdiplomschule } & $\mathrm{F}$ & 2,33 & 1,85 & 2,59 & 1,85 \\
\hline & M & 2,42 & 1,94 & 0,88 & 0,82 \\
\hline \multirow{2}{*}{$\begin{array}{l}\text { Lehrerinnen- und } \\
\text { Lehrerausbildung }\end{array}$} & $\mathrm{F}$ & 1,80 & 2,84 & 3,29 & 3,27 \\
\hline & M & 1,31 & 1,62 & 1,32 & 1,79 \\
\hline \multirow[t]{2}{*}{ «Töchterschule»/DMS } & $\mathrm{F}$ & 2,87 & 3,38 & 3,67 & 3,12 \\
\hline & M & 0,00 & 0,00 & 0,00 & 0,06 \\
\hline \multirow[t]{2}{*}{ Neusprachliches Gymnasium } & $\mathrm{F}$ & - & - & 0,57 & 1,49 \\
\hline & $\mathrm{M}$ & - & - & 0,00 & 0,57 \\
\hline \multirow{2}{*}{$\begin{array}{l}\text { Mittelschülerinnen und } \\
\text { Mittelschüler total }\end{array}$} & $\mathrm{F}$ & 8,82 & 10,46 & 13,84 & 16,64 \\
\hline & M & 9,75 & 10,69 & 11,71 & 13,64 \\
\hline \multirow{2}{*}{$\begin{array}{l}\text { davon Diplommittelschülerinnen } \\
\text { und Diplommittelschüler }\end{array}$} & $\mathrm{F}$ & 7,00 & 8,07 & 9,55 & 8,23 \\
\hline & $\mathrm{M}$ & 3,73 & 3,56 & 2,20 & 2,67 \\
\hline \multirow{2}{*}{$\begin{array}{l}\text { davon Maturitätsmittelschülerinnen } \\
\text { und Maturitätsmittelschüler }\end{array}$} & $\mathrm{F}$ & 1,81 & 2,39 & 4,29 & 8,40 \\
\hline & $\mathrm{M}$ & 6,02 & 7,13 & 9,52 & 10,97 \\
\hline
\end{tabular}

Dieses schnellere Wachstum der Mittelschülerquote der Frauen setzte sich auch nach 1980 fort, sodass diese Ende der 90er-Jahre auch bezüglich der Maturitätsquote die Männer überrundeten.

Dieses Resultat, mit dem niemand gerechnet hatte, warf verschiedenste Planungsannahmen über den Haufen, insbesondere was die Verteilung der Schülerinnen und Schüler auf die verschiedenen Mittelschultypen und in der Folge auf 
die verschiedenen Hochschulfachrichtungen betraf. So konnten letztlich die Nachwuchsprobleme bei den Ingenieuren, welche den Anstoss zur neuen Welle der Mittelschulexpansion gegeben hatten, kaum gelöst werden, da das Problem immer «geschlechtsblind» angegangen worden war.

\section{Soziale Ungleichheit in neuer Bedeutung}

Im Unterschied zu den früheren Jahrzehnten war die Diskussion um den Mittelschulausbau in den 50er- und 60er-Jahren nicht "schichtblind», vielmehr wurde nun bewusst darüber beraten, in welchen sozialen Schichten vermehrt Bildungsreserven ausgeschöpft werden könnten. Entsprechend verbesserte sich auch die Sozialstatistik, sodass der Ausgangspunkt der 2. Welle der Mittelschulexpansion gut dokumentiert ist. Für 1957 verfügen wir über eine statistische Auswertung, welche die soziale Zusammensetzung der Schülerinnen und Schüler verschiedener Mittelschultypen nach Berufsgruppen der Väter wiedergibt (vgl. Tabelle 9). Es zeigt sich eine besonders krasse Untervertretung der Arbeiter- und Bauernkinder am Literar-Gymnasium und eine etwas bessere Vertretung bei den Mittelschulen ohne Hochschulzugang sowie beim an die Sekundarschule anschliessenden mathematisch-naturwissenschaftlichen Gymnasium.

\section{Tabelle 9: Mittelschülerinnen und Mittelschüler nach ausgewählten sozialen} Berufsgruppen der Väter in Prozent aller Schülerinnen und Schüler eines Schultypus, Kanton Zürich, 195715

\begin{tabular}{|l|c|c|c|c|c|c|}
\hline $\begin{array}{l}\text { Berufsgruppen } \\
\text { der Väter }\end{array}$ & $\begin{array}{c}\text { Literar- } \\
\text { Gymn. }\end{array}$ & $\begin{array}{c}\text { Math.- } \\
\text { Gymn. }\end{array}$ & $\begin{array}{c}\text { Lehrerinnen- } \\
\text { und } \\
\text { Lehrerseminar }\end{array}$ & $\begin{array}{c}\text { Handels- } \\
\text { schule }\end{array}$ & $\begin{array}{c}\text { «Töchter- } \\
\text { schule» }\end{array}$ & $\begin{array}{c}\text { alle } \\
\text { Mittelschulen }\end{array}$ \\
\hline Liberale Berufe & 38,5 & 23,7 & 12,3 & 12,7 & 20,9 & 25,1 \\
\hline Beamte/Angest. & 38,2 & 48,0 & 49,2 & 49,4 & 49,5 & 44,5 \\
\hline Bauern & 0,4 & 2,2 & 3,6 & 1,2 & 2,6 & 1,3 \\
\hline Arbeiter & 4,1 & 8,1 & 6,6 & 9,1 & 9,5 & 8,5 \\
\hline Andere & 18,8 & 18,0 & 28,3 & 27,6 & 17,5 & 20,6 \\
\hline Total & 100,0 & 100,0 & 100,0 & 100,0 & 100,0 & 100,0 \\
\hline
\end{tabular}

Nun sagen diese Anteilsdaten noch wenig aus, solange wir sie nicht auf die Grössenverhältnisse der entsprechenden sozialen Gruppen im Kanton Zürich beziehen und Quoten bilden. Dann zeigt sich, dass nur gerade eines von 200 Arbeiterkindern in den 50er-Jahren an eine Maturitätsschule gelangte (vgl. Tabelle 10). Bei den Arbeitermädchen dürfte es sogar nur etwa eines von 250 gewesen sein. 16 
Tabelle 10: Quoten der Mittelschschülerinnen und Mittelschüler nach ausgewählten Berufsgruppen der Väter, Kanton Zürich, 195717

\begin{tabular}{|l|c|c|c|}
\hline $\begin{array}{l}\text { Berufsgruppen } \\
\text { der Väter }\end{array}$ & $\begin{array}{c}\text { Quote Maturitäts- } \\
\text { mittelschülerinnen } \\
\text { und -mittelschüler }\end{array}$ & $\begin{array}{c}\text { Quote Diplommittel- } \\
\text { schülerinnen und } \\
\text { Diplommittelschüler }\end{array}$ & $\begin{array}{c}\text { Quote alle } \\
\text { Mittelschülerinnen } \\
\text { und Mittelschüler }\end{array}$ \\
\hline Arbeiter & 0,4 & 1,3 & 1,7 \\
\hline Bauer & 0,5 & 1,9 & 2,4 \\
\hline Volksschullehrer & 20 & 31 & 51 \\
\hline Arzt & 29 & 12 & 41 \\
\hline Hoch-, Mittelschullehrer & ca. 50 & ca. 20 & ca. 70 \\
\hline
\end{tabular}

Dies war also der Ausgangspunkt, als die soziale Ungleichheit bei der Rekrutierung für die Mittel- und Hochschulen zum Thema der Bildungspolitik wurde. Der Anteil von Arbeiter- und Bauernkindern war dermassen tief, dass die Protagonisten der Mittelschulexpansion mit gutem Grund davon ausgehen konnten, dass hier noch "Begabungsreserven» zu entdecken wären. Ein Vergleich mit dem Ausland zeigte schon damals, dass höhere Anteile von Arbeiterkindern an der höheren Ausbildung möglich waren: Nur gerade Deutschland hatte vergleichbar tiefe Werte wie die Schweiz, während Länder wie Frankreich und England bereits um einiges höheere Arbeiterkinder-Anteile unter den Studierenden aufwiesen.

Tabelle 11: Studentenanteile nach sozialer Stellung der Väter, Europa um 1960 (Kälble, 1983, S. 214 f.)

\begin{tabular}{|l|c|c|}
\hline & $\begin{array}{c}\text { Anteil Studierende mit } \\
\text { Arbeiter-Vätern }\end{array}$ & $\begin{array}{c}\text { Anteil Studierende mit } \\
\text { freiberuflich tätigen Vätern }\end{array}$ \\
\hline Schweiz & $6 \%$ & $24 \%$ \\
\hline Italien & $13 \%$ & $12 \%$ \\
\hline Schweden & $16 \%$ & $21 \%$ \\
\hline England & $23 \%$ & - \\
\hline Deutschland & $5 \%$ & $10 \%$ \\
\hline Dänemark & $9 \%$ & $31 \%$ \\
\hline
\end{tabular}

Welche Auswirkungen hatten nun zwei Jahrzehnte der Mittelschulexpansion und der «Begabtenförderung» in unteren sozialen Schichten? 
Tabelle 12: Prozentanteil von Arbeiterkindern an allen Mittelschülerinnen und Mittelschülern eines Schultypus, Kanton Zürich, 1957/58-1980

\begin{tabular}{|l|c|c|c|}
\hline & $1957 / 58$ & 1969 & 1980 \\
\hline $\begin{array}{l}\text { Literar-Gymnasium } \\
\text { Langzeitgymnasium } \\
\text { Kurzzeitgymnasium }\end{array}$ & 4,1 & 5,7 & \\
\cline { 2 - 4 } & & & 15,9 \\
\cline { 2 - 4 } $\begin{array}{l}\text { Mathematisch-naturwissenschaftliches- } \\
\text { Gymnasium }\end{array}$ & 8,1 & 13,5 & 15,9 \\
\hline $\begin{array}{l}\text { Handelsmittelschule } \\
\text { Handelsmaturitätsschule }\end{array}$ & 9,1 & 12,0 & \\
Handelsdiplomschule & & 10,0 & 11,0 \\
\hline \begin{tabular}{l} 
Lehrerinnen- und Lehrerseminar \\
\cline { 2 - 4 }
\end{tabular} & 16,6 & 14 & 16,5 \\
\hline «öchterschule»/DMS & 9,5 & 11,1 & 17,4 \\
\hline alle Mittelschultypen & ca. 9 & ca. 10 & ca. 12 \\
\hline
\end{tabular}

Wie Tabelle 12 zeigt, stieg der Anteil Arbeiterkinder am Gesamt der Mittelschülerinnen und Mittelschüler von rund 9 auf 12 Prozent, wobei diese Zunahme nicht wie erwartet beim Lehrerseminar und der Handelsmittelschule erfolgte, sondern bei den Kurzzeitgymnasien (beim mathematisch-naturwissenschaftlichen Gymnasium und beim neusprachlichen Kurzzeitgymnasium).

Was sind nun die entsprechenden Quoten bezogen auf ganze Schülerjahrgänge und unter Einbezug der Tatsache, dass der Anteil Arbeiterväter am Gesamt der Väter bis 1980 kleiner geworden ist?

Tabelle 13: Quoten des Mittelschulbesuchs bei Arbeiterkindern, Kanton Zürich, 1958-1980

\begin{tabular}{|l|c|c|}
\hline & $\begin{array}{c}\text { Quote alle } \\
\text { Mittelschulen }\end{array}$ & $\begin{array}{c}\text { Quote } \\
\text { Maturitätsschulen }\end{array}$ \\
\hline 1958 & $1,8 \%$ & $0,5 \%$ \\
\hline 1969 & $3,0 \%$ & $1,1 \%$ \\
\hline 1980 & $4,7 \%$ & $3,3 \%$ \\
\hline 1985 & $5,8 \%$ & $3,8 \%$ \\
\hline
\end{tabular}

Arbeiterkinder besuchen 1980 - wie Tabelle 13 ausweist - mit etwa 8-mal grösserer Wahrscheinlichkeit eine Maturitätsschule als noch 1958 und 3-mal wahrscheinlicher eine Mittelschule. Dies bedeutet zweifellos einen Fortschritt in Richtung der intendierten Förderung von «Begabungsreserven» in unteren Schichten.

Allerdings hat sich unterdessen das Gesamtbild sehr stark verändert. Zum einen hat sich die Maturitätsschulquote im gesamtgesellschaftlichen Durchschnitt ebenfalls mehr als verdoppelt (von 4 Prozent 1939 auf 10 Prozent 1980). Zum 
andern haben andere gesellschaftliche Gruppen ihre Partizipation an den Mittelschulen ebenfalls gesteigert.

1985, zum letzten Zeitpunkt, als noch differenzierte Berufsdaten veröffentlicht wurden, zeigt sich bei einigen ausgewählten Berufen (Berufe, deren Kategorien in der Schulstatistik mit denen in der Volkszählung vergleichbar sind) folgendes Bild:

Tabelle 14: Mittelschülerquoten nach Beruf der Väter, Kanton Zürich, 1985

\begin{tabular}{|l|c|c|}
\hline Beruf des Vater & $\begin{array}{c}\text { Mittelschüler- } \\
\text { Quote der Kinder }\end{array}$ & $\begin{array}{c}\text { Maturitätsschüler- } \\
\text { Quote der Kinder }\end{array}$ \\
\hline Maler & 4,8 & 3,3 \\
\hline Bauer & 7,2 & 4 \\
\hline Drucker & 9,1 & 6,5 \\
\hline Polizist & 15 & 10,4 \\
\hline Arzt & 60 & 49 \\
\hline Hoch-/Mittelschullehrer & 85 & 64 \\
\hline Volksschullehrer & 67 & 40 \\
\hline
\end{tabular}

In der Relation zur Entwicklung des Mittelschulbesuchs privilegierter Gruppen bleibt der Fortschritt bei Kindern unterer sozialer Schichten also sehr bescheiden. Die Abstände der sozialen Positionen haben sich damit nicht verringert.

War eines der Ziele der «Nachwuchsförderung» die vermehrte Rekrutierung von Arbeiter- und Bauernkindern, so ist dies nur in kleinem Ausmass gelungen. Der Grossteil der durch die Bildungsexpansion in absoluten Zahlen zusätzlich Rekrutierten stammt keineswegs aus diesen beiden sozialen Gruppen, die 1985 immer noch nur 14 Prozent aller Mittelschülerinnen und Mittelschüler, aber fast 40 Prozent der erwerbstätigen Väter stellen. Der Grossteil der zusätzlich für die Mittelschule Rekrutierten stammt vielmehr aus den mittleren und oberen Angestellten- und Beamtenschichten.

Neu hinzu kommt aber auch eine Veränderung des Bildungsverhaltens in der Oberschicht, die bis in die 50er-Jahre abgesehen vom «Bildungsbürgertum» keineswegs stark auf eine akademische Ausbildung ihrer Kinder ausgerichtet war.

Über dieses mehr als bescheidene Resultat der Nachwuchsförderung in unteren sozialen Schichten wird in den 80er-Jahren im Kanton Zürich kaum Bilanz gezogen. Eine Ausnahme machen Bernath et al., welche ihre Kohortenuntersuchung eines Schülerjahrganges bis in die 80er-Jahre weiterführten und zu deutlichen Resultaten kamen (vgl. Tabelle 15). 
Tabelle 15: Laufbahnpositionen von Jugendlichen aller Schichten mit 21 Jahren, Kanton Zürich, 1982 (Bernath et al., 1989, S. 67)

\begin{tabular}{|l|c|c|c|}
\hline Laufbahnposition & $\begin{array}{c}\text { Jugendliche aus der } \\
\text { Unterschicht }\end{array}$ & $\begin{array}{c}\text { Jugendliche aus der } \\
\text { Mittelschicht }\end{array}$ & $\begin{array}{c}\text { Jugendliche aus der } \\
\text { Oberschicht }\end{array}$ \\
\hline $\begin{array}{l}\text { Maturitäts-/Hochschule } \\
\text { Höhere Fachschule }\end{array}$ & $6,0 \%$ & $20,5 \%$ & $40,5 \%$ \\
\hline Erwerbstätig & $70,0 \%$ & $51,5 \%$ & $29,0 \%$ \\
\hline
\end{tabular}

Solche Befunde vermochten jedoch - im Gegensatz zu den 60er- und 70er-Jahren - keine öffentliche Diskussion über die Chancenungleichheit im Bildungssystem mehr auszulösen, ja das Thema wurde offensichtlich verdrängt. ${ }^{18}$ Dies ist umso unverständlicher, als gerade ab den 80er-Jahren die bescheidenen Fortschritte in der Mittelschulpartizipation von Kindern unterer Schichten - im Unterschied zu Kindern aus mittleren und oberen Schichten der Gesellschaft - ins Stocken geraten sind, wenn sie nicht gar rückläufig wurden (Huber, 1993, S. 16 f.).

\section{Ausländerkinder: Entscheidende Schichtzugehörigkeit}

Hohe Immigrantenanteile in den Schulen des Kantons Zürich wurden bereits seit Ende des 19. Jahrhunderts konstatiert, aber nicht systematisch erhoben. Genaueres über die Bildungsbeteiligung der Ausländerkinder wissen wir erst seit Mitte der 60er-Jahre. Dennoch bleibt es im Mittelschulbereich besonders schwierig, ausgehend von den vorhandenen Daten Aussagen zu machen. Zwar kennen wir seit 1968 die Anzahl Mittelschülerinnen und Mittelschüler nach Nationalität, aber wir wissen nicht, auf welche Grössen wir diese Daten beziehen müssen.

Für den Kanton Zürich hat eine Kohorten-Untersuchung den schulischen Werdegang eines Schuljahrganges von Italienerinnen und Italiener verfolgt und mit einer Kontrollgruppe von Schweizerinnen und Schweizern verglichen (Gurny et al., 1984). Dabei wurde der enge Zusammenhang mit der sozialen Herkunft deutlich. Wird nämlich die präzise soziale Position der Väter analysiert (und zwischen Vorarbeitern, gelernten und ungelernten Arbeitern differenziert), nähert sich die Bildungspartizipation der italienischen Jugendlichen jener der entsprechenden Schweizer Jugendlichen an:

Tabelle 16: Erreichte Schulstufe von Jugendlichen der untersten sozioökonomischen Schicht, Zürich, 1981 (Gurny et al., 1984, S. 80)

\begin{tabular}{|l|c|c|}
\hline & $\begin{array}{c}\text { Jugendliche italienischer } \\
\text { Nationalität }\end{array}$ & $\begin{array}{c}\text { Jugendliche mit Schweizer } \\
\text { Nationalität }\end{array}$ \\
\hline Sekundarschule & $37 \%$ & $41 \%$ \\
\hline Realschule & $48 \%$ & $50 \%$ \\
\hline Oberschule/Sonderschule & $15 \%$ & $9 \%$ \\
\hline
\end{tabular}


Ein grösserer Unterschied zwischen italienischer und schweizerischer Herkunft bei konstant gehaltener Sozialschicht ist also nur bei den Schülerinnen und Schülern des untersten Schulniveaus festzustellen, während bei der Mehrheit die Schüleranteile relativ parallel verlaufen. Untersuchungen in anderen Kantonen sind zu ähnlichen Resultaten gekommen (Hutmacher, 1987).

Diese Resultate sind bemerkenswert, werden doch in der Diskussion immer wieder die besonderen kulturellen Integrationsprobleme der Immigrantenkinder als Hauptgrund für ihre schulischen Schwierigkeiten hervorgehoben.

\section{Die Erosion des katholischen Milieus und der besonderen Bildungslaufbahn}

Bei der katholischen Minderheit, welche sich in Zürich in einem kulturellen "Gegenmilieu» zu organisieren versuchte, hatten wir bis in die 50er-Jahre ein ganz besonderes Muster des Mittelschulbesuchs ausgemacht, nämlich den Besuch von Internaten im katholischen «Hinterland».

Seit den 60er-Jahren wird dieses Muster immer schwächer. Dies hat primär sicher damit zu tun, dass sich das katholische Gegenmilieu selbst auflöste und damit auch seine normenbildende Kraft. Die Katholiken in Zürich wandelten sich von einer sich diskriminiert fühlenden Minderheit zu einer normalen anerkannten Gruppe in der Gesellschaft: Ihre Partei wurde neu in den Regierungsrat aufgenommen; die Katholische Kirche wurde bezüglich Einzug der Kirchensteuer der Protestantischen gleichgestellt; ein Teil der Katholiken erreichte schliesslich im Rahmen der wirtschaftlichen Nachkriegs-Hochkonjunktur auch einen sozialen Aufstieg. Auf der Gegenseite legten die Freisinnigen ab Mitte der 60er-Jahre ihren Antiklerikalismus beiseite. Im Rahmen des Ausbaus der Mittelschulen fanden nun auch Mittelschullehrerinnen und Mittelschullehrer mit katholischem Taufschein Anstellung in Zürichs Schulen. In der Folge begann nicht nur der Aderlass der Vereine des katholischen Milieus und der Abonnentinnen und Abonnenten der katholischen Tageszeitung «Neue Zürcher Nachrichten», in der Folge stockte auch der Fluss katholischer Jugendlicher in Richtung der Internate. Mehrere dieser Institutionen wurden aufgelöst (Altdorf, Stans ...), oder aber sie überlebten nur dank Umwandlung in öffentliche, geschlechtsgemischte Schulen mit regionalem Einzugsgebiet.

Waren die früheren besonderen konfessionellen Schülerströme nie quantitativ untersucht worden, so kennen wir das Resultat des Prozesses am Ende der zweiten grossen Welle der Bildungsexpansion: Im Rahmen ihrer Kohortenuntersuchung hatten Bernath et al. (1980) auch die Konfessionszugehörigkeit erhoben und festgestellt, dass sich «hinsichtlich der Konfessionszugehörigkeit praktisch eine Nullkorrelation» (Bernath et al., 1980, S. 93) ergibt. Die katholischen Eltern respektive die katholischen Jugendlichen verhielten sich also spätestens seit den 80er-Jahren - natürlich je nach ihrer Schichtzugehörigkeit, dem Geschlecht ihrer Kinder, etc. - in Bezug auf den Mittelschulbesuch nicht mehr anders als die entsprechenden protestantischen Eltern respektive die protestanti- 
schen Jugendlichen - auch dies eine gelungene, wenn auch in der offiziellen bildungspolitischen Zielsetzung nie intendierte Angleichung der sozialen Chancen im Laufe der zweiten Welle der Bildungsexpansion an den Zürcher Mittelschulen.

\section{Zusammenfassende Interpretation}

In den 80er-Jahren konnte also als Resultat der Bildungsexpansion für die Mittelschulen positiv festgestellt werden:

- Die Mittelschülerzahlen haben sich wie geplant um ein Mehrfaches ausgeweitet, der Nachwuchs-Bedarf der Wirtschaft konnte im Grossen und Ganzen gedeckt werden.

- Der alte Gegensatz von Stadt und Land ist aufgelöst.

- Die konfessionsspezifischen Bildungsmuster haben sich verflüchtigt, die Katholiken klagen nicht mehr über Diskriminierungen.

- Die Frauen haben nicht nur bei den Diplommittelschulen mit den Männern gleichgezogen, respektive sie überrundet, sondern auch bei den Gymnasien.

- Bloss bei einer Ungleichheitsbeziehung hat die Bildungsexpansion letztlich grundlegend nichts geändert: bei der schichtspezifischen Partizipation an höherer Bildung.

Anders gesagt: Die Klage über die Diskriminierung des katholischen Arbeitermädchens vom Lande war passée. Es bleibt aber nach wie vor Grund zur Klage über die Diskriminierung der Kinder unterer sozialer Schichten.

Die erfolgreichen Elemente der Entwicklung sind gut erklärbar:

- Die Überwindung des Stand-Land-Gegensatzes wurde grundlegend ermöglicht durch strukturelle Durchmischung und Agglomerierung von Siedlungen und Betrieben, aber auch der Wohnsitze von Mittel- und Oberschichten.

- Die Auflösung der besonderen Bildungsmuster der Zürcher Katholiken geht einher mit der Auflösung des katholischen Sozialmilieus im Zuge des sozialen Aufstiegs eines Teils der katholischen Minderheit und ihrer Integration in den Zürcher «Bürgerblock».

- Das bildungsmässige Gleichziehen der Frauen setzte Jahrzehnte von Kämpfe gegen die patriarchalen Barrieren in den Schulen, aber auch innerhalb der Familien voraus. Dieser Prozess wurde unterstützt durch den sich verändernden Arbeitskräftebedarf mit der Tertiarisierung der Wirtschaft, speziell durch den vermehrten Bedarf in den Bereichen des Sozial-, Erziehungs- und Gesundheitswesens.

Warum jedoch der Misserfolg bezüglich der unteren sozialen Schichten? Grundlegend dafür ist sicher die Tatsache, dass sich die schicht- oder klassenmässige Strukturierung der Gesellschaft in den Nachkriegsjahrzehnten in der Schweiz nicht aufgelöst hat (vgl. dazu u.a. Levy, 1997). Beim Zugang zu den höheren Ausbildungen bleiben damit für Kinder aus unteren sozialen Schichten 
viele der Barrieren aufrechterhalten, welche in den 60er- bis 80er-Jahren vielfach untersucht und konstatiert worden waren:

- Die hemmende Wirkung finanzieller Barrieren auf den Mittelschulbesuch unterer Schichten ist offensichtlich. Sie wurde durch bessere Stipendien und die Abschaffung respektive Absenkung von Schulgeldern nur zum Teil abgebaut. Bereits seit Ende der 70er-Jahre wurden die Stipendien wieder restriktiver geregelt und es wurden wieder Gebühren eingeführt oder erhöht. Bildungsökonomisch hat sich zudem gezeigt, dass individuelle finanzielle Investitionen in höhere Ausbildung durchaus unsicherer Natur sind (Wolter, 1994), was je nach finanziellem Hintergrund der Eltern natürlich unterschiedliche Folgen für die Bildungsentscheidungen hat.

- Die Bildungsaspirationen der Eltern haben einen entscheidenden Einfluss auf die Chancen der Kinder und sind nach sozialer Herkunft sehr ungleich. Ihre hemmende Wirkung auf den Mittelschulbesuch wird verstärkt durch die schichtabhängige Beurteilung der Mittelschulreife von Kindern und Jugendlichen durch die Lehrerinnen und Lehrer (vgl. für den Kanton Zürich die Untersuchungen von Haefeli et al., 1979; Karrer, 2000; Müri, 1971; Trier, 1964).

- Die kulturelle Distanz zum - bildungsbürgerlich oder zumindest mittelständisch geprägten - Milieu der Mittelschulen wurde schliesslich als weitere wichtige Barriere für Kinder aus unteren sozialen Schichten immer wieder hervorgehoben (vgl. u.a. Bourdieu \& Passeron, 1991; Fend, 1981; Hess et al., 1966).

Diese Barrieren sind struktureller Natur. Welche Wirkung sie entfalten, ist aber auch davon abhängig, ob eine bewusste Bildungspolitik die Barrieren zu überwinden oder zu kompensieren versucht. Sei es, um genügend hoch qualifizierte Arbeitskräfte nachzuziehen, sei es aus Gerechtigkeitsempfinden engagierten sich seit Ende der 50er- bis anfangs der 70er-Jahre viele Politikerinnen und Politiker und pädagogisch Tätige für eine vermehrte Partizipation der Unterschichten an den Mittelschulen. Sie haben die öffentliche Meinung während einer Periode geprägt, Massnahmen zum Abbau respektive zur Kompensation von Bildungsbarrieren vorgeschlagen und umgesetzt. Dass es sich bei der Verfolgung des Ziels von mehr Chancengleichheit nicht um eine reine Illusion handelte, konnte in Zwischenbilanzen Ende der 60er-Jahre in Zürich festgestellt werden, aber auch im Vergleich mit anderen Ländern, wo die Mittelschulquoten der Unterschichten zwar nie jene der Oberschichten erreichten, aber doch die Werte der Schweiz weit übertrafen. Die Massnahmen, welche bewusst zwecks Erleichterung der Mittelschulpartizipation der Unterschichten umgesetzt wurden, blieben im Kanton Zürich allerdings weitgehend auf den Abbau von materiellen und psychologischen Barrieren beschränkt. Reformen der Sekundarstufe I in Richtung Gesamtschule wurden in Zürich nur von der SP und einer Minderheit von Bildungsverantwortlichen vertreten (Häberlin, 1972), ganz im Unterschied zum Kanton Genf oder zu einzelnen Bundesländern Deutschlands (Fend, 1982). 
Seit Ende der 70er-Jahre verstummte die Diskussion um die Chancengleichheit in der Öffentlichkeit. Die bildungspolitische Diskussion, die im Kanton Zürich zu verschiedenen Fragen durchaus rege geführt wurden (Reform der Sekundarstufe I, Integration von Immigrantenkindern, New Public Management, Entwicklung der Fachhochschulen etc.) verlief seit dieser Zeit schichtblind. Dies gilt weitgehend auch für die sozialdemokratische Schulpolitik.

Dabei hätten die auch für Zürich durchaus vorhandenen bildungswissenschaftlichen Befunde eine erneute Diskussion über den ungleichen Zugang zur Bildung nahegelegt. Die bescheidene Zunahme der Mittelschulbeteiligung von Unterschichtskindern wurde deutlich relativiert durch die massive Zunahme der Mittel- und Oberschichtkinder. Gleichzeitig erschienen Studien, welche für die Bundesrepublik Deutschland weit höhere Abiturquoten von Arbeiterkindern in Bundesländern mit Gesamtschulen nachwiesen (Fend, 1982). Warum vermochte dies im Kanton Zürich keine erneute gesellschaftliche Diskussion mehr auszulösen?

Der wichtigste Grund liegt sicher darin, dass Arbeitgeberorganisationen und bürgerliche Parteien im Unterschied zu den 50er- und 60er-Jahren das Interesse an dieser «Nachwuchsförderung» verloren: Der nötige Nachschub an höher qualifizierten Arbeitskräften schien gedeckt zu sein. Häufiger wurden nun - auf dem Hintergrund der neoliberalen Renaissance und der Sparpolitik der öffentlichen Hand - Diskussionen über die «individuelle Selbstverantwortung» für die nachobligatorische Bildung geführt.

Warum wurde aber die fortgesetzte Schicht-Ungleichheit auch in "fortschrittlichen» Kreisen kaum mehr wahrgenommen?

Die erfolgreiche Auflösung der ungleichen Partizipation von Frauen, Kindern vom Lande und aus katholischen Familien konnte dazu verführen, den kleinen Fortschritt bei den Unterschichtskindern als erfolgsversprechenden Beginn eines - etwas langsameren - Prozess auch bei den Kindern aus der Unterschicht zu interpretieren. Dazu hat die in breiten Kreisen rezipierte Individualisierungsthese von Ulrich Beck (Beck, 1983, 1986) einen nicht unwesentlichen Beitrag geleistet. Diese konnte die Auflösung «ständischer» Beschränkungen bezüglich Bildung bei der ländlichen Bevölkerung, bei den Frauen und bei den Katholikinnen und Katholiken bestens beschreiben. Auch die Auflösung des organisierten Sozialmilieus der Arbeiterschaft wird zu Recht konstatiert, dann jedoch missverstanden als Auflösung der kulturellen Klassenmilieus an sich, indem behauptet wird, "dass durch Niveauverschiebungen (Wirtschaftsaufschwung, Bildungsexpansion) subkulturelle Klassenidentitäten zunehmend weggeschmolzen [...] und Prozesse einer Diversifizierung und Individualisierung von Lebenslagen ausgelöst wurden, die das Hierarchiemodell sozialer Klassen und Schichten [...] in seinem Realitätsgehalt zunehmend in Frage stellen» (Beck, 1983, S. 36). Dies führe verbunden mit der Bildungsexpansion zu einer "Flucht der Arbeiterkinder aus der Arbeiterschaft» (Beck, 1983, S. 38), die Herauslösung aus dem Herkunftsmilieu werde «zum selbstverständlichen Massenschicksal»(Beck, 1983, S. 45). Dieser 
Prozess schlage sich nieder in einer «deutlichen Verschiebung in den Ungleichheitsrelationen» bei der Bildung (Beck, 1986, S. 127).

Empirisch waren und sind diese Thesen nicht haltbar, weder bezüglich der Auflösung kultureller Milieus mit sozio-ökonomischem Hintergrund (Karrer, 2000), noch bezüglich Auflösung der Schichtungleichheit im Zuge der Bildungsexpansion, wie in diesem Aufsatz für Zürich nachgewiesen wird oder Mayer (1990) für Deutschland zeigte. Dennoch hatten sie ein riesiges Echo.

Aus dem Blickfeld geriet mit der Abwendung von Klassentheorien auch die Frage, wie sich die sozialen Schichten und Milieus am oberen Ende der sozialen Hierarchie entwickelten. So ist gerade in Bezug auf die Bildungspartizipation eine ganz wichtige Veränderung fast unbemerkt geblieben:

Bis in die 50er-Jahre galt eine höhere schulische Ausbildung nur im akademischen Bildungsbürgertum als soziale Norm, jedoch überhaupt nicht beim Besitzbürgertum: Dessen Söhne brauchten überhaupt keine Matura, sondern einen guten Sekundarschulabschluss, einen kaufmännischen Lehrabschluss oder einen Abschluss einer Diplommittelschule, und in der Folge eine individuelle Ausbildung mit Praktika «von der Pike auf» bei Geschäftsfreunden im In- und Ausland oder im eigenen Betrieb. Die Spitze der Schweizer Wirtschaft blieb so bis in die Gegenwart sehr wenig akademisiert, was sich in den Biographien vieler schweizerischer "Wirtschaftskapitäne» nachlesen lässt, die in diesen Jahren in Pension gehen. Die Volkszählung weist für 1990 bei den 60- bis 64-jährigen schweizern im obersten Management einen Anteil von nur gerade 30\% Hoch- und Fachhochschulabschlüsse aus. In dieser Hinsicht unterschieden sich die Schweizer Unternehmer bis vor kurzem deutlich von den meisten anderen Ländern, deren oberste Wirtschaftskader seit langem mehrheitlich akademisiert waren (Kälble, 1983, S. 240 ff.).

In der zweiten Welle der Bildungsexpansion hat sich die Zürcher Unternehmerschicht vom bisherigen Laufbahnmustern verabschiedet, ist doch nun der Mittelschulbesuch zur Norm geworden. So weist die Oberschicht der Gemeinde Zollikon heute eine Mittelschulquote von gegen $80 \%$ auf. ${ }^{19}$ Mit Verzögerung beginnt dies für die Bildungsstruktur in den Führungsetagen der Grossunternehmen Folgen zu haben (vgl. Weber, 1985, S. 84 ff.), die noch der genaueren Untersuchung harren. ${ }^{20}$

Diese Veränderung in der Oberschicht ist in ihrer Bedeutung für die Ungleichheitsrelationen bisher nicht genügend gewertet worden. ${ }^{21}$ Werden höhere Schulabschlüsse nun zur notwendigen Voraussetzung, um in die Führungsetagen zu gelangen, und verfügt nur eine kleine Minderheit der Unterschicht darüber, dann verschliessen sich früher vorhandene Wege der Aufwärtsmobilität.

Die Bildungsexpansion führt so zu einem äusserst paradoxen Resultat für die unteren sozialen Schichten: Sie hat für Arbeiterkinder den fast gänzlichen Ausschluss von Maturitätsschulen aufgebrochen und zu einer durchaus realistischen, aber mit 5\% sehr kleinen Wahrscheinlichkeit des Zugangs zum Gymnasium geführt. Ist damit bewiesen, dass auch Arbeiterkinder «Chancen» haben, dann 
kehrt sich dies gleich gegen sie: Die Realisierung der Chance wird zum «scheinbar individuell lösbaren Bildungsproblem» (Heid, 1988, S. 7). Das Konzept der Chancengleichheit kann so zum Bumerang werden. "Die Forderung nach Chancengleichheit beinhaltet wohl eine Kritik an bestehende Ungleichheiten und ihre Durchsetzung garantiert eine nicht zu unterschätzende formale Gleichheit, nach der niemand von vorneherein ausgeschlossen werden darf, gleichzeitig hat Chancengleichheit als Wettbewerbsformel Ungleichheit als Voraussetzung und als Ergebnis» (Lamprecht, 1991, S. 152).

Die Folgen der Ungleichheit werden umso deutlicher, wenn nicht allein die Partizipation an den Mittelschulen im Blickfeld steht, sondern auch die gleichzeitige schichtspezifische Entwicklung der Sekundarstufen I und II insgesamt und speziell diejenige der Berufsbildung. Ein alleiniger Abschluss der Volksschule, aber auch einer Berufslehre hat in der Zwischenzeit an Wert verloren und eröffnet geringere berufliche Chancen als noch früher ${ }^{22}$. Statt einer Abschwächung der Bildungsungleichheiten resultiert damit insgesamt eher eine erneute «Polarisierung». «Dadurch, dass sich bei den un- und angelernten Arbeitern und in einem gewissen Masse bei den minder qualifizierten Facharbeitern [...] die primären Herkunftseffekte weiterhin ungünstig auf den Bildungsverlauf auswirken, hat sich die Situation der ohnehin benachteiligten Kinder eher verschlechtert als verbessert. Bei der Bildungsbeteiligung haben sich die Abstände zwischen den privilegierten und benachteiligten Sozialschichten vergrössert» (Becker, 2000, S. 466).

In Abwandlung der oben zitierten Aussagen von Ulrich Beck könnte man pointiert formulieren: Das «Massenschicksal» der Unterschichtskinder bleibt die obligatorische Volksschule, ergänzt mit einer Berufslehre, während die Oberschichtskinder die Berufslehre meiden und in die Mittelschulen "fliehen", um den Status ihres Herkunftsmilieus zu erhalten.

Auch wenn sich die Herstellung von wirklicher "Chancengleichheit» im Bildungswesen also bisher als "Illusion» (Bourdieu \& Passeron, 1971) erwies, so entbindet dies nicht von der Kritik der real existierenden Ungleichheit in der Bildung und soll auch nicht zu fatalistischen oder zynischen Haltungen führen. Unterschiedliche Politiken führen zu durchaus unterschiedlichen Ungleichheitsrelationen. Dies kann der Boden dafür sein, dass die unterprivilegierten Schichten und ihre Interessensorganisationen den nach wie vor "plutokratisch» geprägten Zugang zu den Mittelschulen in Zukunft wieder vermehrt in Frage stellen dies umso mehr, wenn sich ein Mittelschulabschluss als wesentlich notwendige (wenn auch nicht hinreichende) Bedingung für immer mehr höhere Positionen in der Gesellschaft erweist. 


\section{Anmerkungen}

1 Nach Gretler (2000, S. 130 f.) war die Ausschöpfung der Begabungsreserven und die Chancengleichheit eines der beiden Hauptthemen der Bildungspolitik und Bildungsforschung der ersten Nachkriegsjahrzehnte. Dies gilt auch für den Kanton Zürich, wo mehrere Untersuchungen zu diesen Fragen durchgeführt wurden (Müri, 1971; Trier, 1964).

2 Für die Berechnungen der Mittelschüler-Quoten wird jeweils das 10. und 11. Schuljahr betrachtet, da zu diesem Zeitpunkt die Ausbildung an allen Mittelschultypen begonnen hat und noch keine abgeschlossen ist.

3 Der Artikel ist Teil einer umfassenderen Untersuchung, welche auch die Primarstufe, die Sekundarstufe I sowie die Berufsbildung umfasst; sie liegt allerdings nur in Form von unveröffentlichten Manuskripten vor (Rieger, 1992).

4 Die Bildungsstatistik bestand während Jahrzehnten aus einzelnen Schulstatistiken, welche die Anzahl der Schülerinnen und Schüler sowie der Klassen auswies und darin Prozentanteile einzelner Gruppen. So wurden z.B. 1957 Anteile von Arbeiterkindern an den Mittelschülern von $8 \%$ und Anteile von Kindern mit freiberuflich tätigen Vätern von 25\% ausgewiesen. In der vorliegenden Untersuchung wurden wenn immer möglich Quoten errechnet, d.h. die Anzahl einzelner Schülergruppen auf die gesamten entsprechenden Schülerjahrgänge resp. die entsprechende Teilgruppe bezogen. So wird die Wahrscheinlichkeit, die Mittelschule zu besuchen, innerhalb einer Jahrgangsgruppe sichtbar. Für die Arbeiterkinder liegt beim obigen Beispiel die Quote bei 1,7\%, bei den Kindern von freiberuflich Tätigen dagegen bei $40 \%$ und mehr (vgl. Tabellen 9 und 10).

5 Quelle: eigene Berechnungen auf Basis der kantonalen Schulstatistiken.

6 Die Kapazitäten der Lehrerseminarien wurden seit längerem "planwirtschaftlich» gesteuert, resp. übersteuert (Schmid, 1982, S. 111).

7 Quellen: Statistische Jahrbücher des Kantons Zürich 1900, 1930, 1950 und entsprechende Mittelschülerstatistik.

8 Ohne grosse Auswirkungen blieben auch die Tiraden einiger Politiker gegen das «Doppelverdienertum» und die Abwehrhaltung der Gesellschaft Schweizer Akademiker, welche 1939 das Anwachsen der Anzahl erwerbstätiger Akademikerinnen als «Unglück» bezeichnete (zitiert nach Dünki, 1985, S. 24).

9 Erste Statistiken für die höhere Bildung bestehen auf schweizerischer Ebene seit 1935, allerdings noch nicht für Mittelschülerinnen und Mittelschüler, sondern für Studierende (Eidgenössisches Statistisches Amt, 1938, 1947, 1961). Für die Universität Zürich wurde vom Autor als Vergleichserhebung für 1945-50 die Immatrikulationsstatistik ausgewertet (Die Studierenden an der Universität Zürich, 1953).

10 Eigene Berechnungen auf der Basis von: Eidgenössisches Statistisches Amt, 1936.

11 Mündliche Auskunft an den Autor von verschiedenen Gewährspersonen des früheren «Katholisch Zürich».

12 Für die - zum Teil im Nebenamt - tätigen Richter ist im Kanton Zürich formell weder ein akademischer Abschluss noch eine entsprechende Prüfung Voraussetzung. In der Praxis wurde dieses Amt auf allen Ebenen bis in die 70er-Jahre des 20. Jahrhunderts auch von Personen ohne höhere Ausbildung ausgeübt.

13 Eine überproportionale Zunahme der Frauen an den Gymnasien wurde in keinem der massgebenden Planungsberichte der Erziehungsbehörden überhaupt nur in Erwägung gezogen, was einen guten Teil der Fehlprognosen erklärt.

14 Ein Artikel von 1962, der die Rekrutierungsschwierigkeiten der Eidgenössisch Technischen Hochschule (ETH) schilderte, widmet der Frage, wie möglicherweise auch Frauen für die ETH gewonnen werden könnten, keine Zeile (Vogt, 1962). Die Volkszählung von 1960 weist für den Kanton Zürich 5 Maschineningenieurinnen aus, allesamt sind sie Ausländerinnen. Frauen in diesem Beruf gab es also, aber keine Diskussion darüber! 
15 Quelle: Statistische Berichte (1959, S. 98) und eigene Berechnungen.

16 Die Daten sind leider nicht nach Geschlecht zugänglich. Aus der gesamtschweizerischen Befragung von 1959 wissen wir jedoch, dass die Quote der Arbeiter-Mädchen damals etwa halb so gross war wie jene der Arbeiter-Knaben.

17 Quelle: Eigene Berechnungen nach Volkszählung (1950) und Statistische Berichte (1959).

18 Seit 1968 waren im Kanton Zürich systematisch Schichtmerkmale für die Mittelschulstatistik erhoben und publiziert worden. 1986 stellte die Erziehungsdirektion die Publikation der Schichtdaten ein, seit 1996 werden sie auch nicht mehr erhoben.

19 Die Mittelschülerquote betrug in Zollikon 1990 insgesamt 74\%. Nun besteht die Zolliker Bevölkerung aber keineswegs nur aus Oberschicht, im Gegenteil weist die Volkszählung einen nicht zu vernachlässigenden Anteil von Arbeitern, unteren Angestellten und anderen aus, deren Mittelschülerquote sicher wesentlich unter $74 \%$ liegt.

20 Von den 35- bis 39-jährigen Direktoren verfügten gemäss Volkszählung noch 1990 nur $35 \%$ über einen Hochschul- oder einen Fachhochschulabschluss. Die Bildungsentscheide dieser Altersgruppe wurden jedoch noch vor der vollen Entfaltung der Bildungsexpansion getroffen. Deren Effekt auf die Akademisierung des obersten Kaders dürfte erst in der Volkszählung 2000 sichtbar werden. Eine Untersuchung von B. Liebig weist bereits für 1994 für enger definierte Unternehmenskader einen 80\%-Anteil von Tertiärabschlüssen aus (Liebig, 1997, S. 131).

21 Lamprecht geht davon aus, dass die Bildungsexpansion eine vermehrte Teilhabe der Unter- und Mittelschichten an der Mittelschule erlaubte, ohne die Chancen der Oberschicht zu «schmälern» (Lamprecht, 1991, S. 152). Es ist jedoch vielmehr so, dass sich die Mittelschulpartizipation der Oberschicht grundlegend ausgeweitet hat. Auch Weber (1985) erfasst die Bedeutung der neuen Entwicklung nicht, wenn er schreibt: «Eine akademische Ausbildung wird weiterhin (Hervorhebung AR) eine notwendige, jedoch immer weniger eine hinreichende Bedingung für den Zugang zu Elitepositionen» (84). Bis in die 80erJahre war eine akademische Ausbildung gerade keine notwendige Bedingung für die meisten Elitepositionen in der Schweiz.

22 Was die Berufsbildungsreform mit der Einführung der Berufsmatura für die Entwicklung der schichtspezifischen Chancen bedeutet, ist meines Wissens in der Deutschschweiz bisher weder diskutiert noch erforscht.

\section{Literatur}

Aebli, D. (1963). Aus der Sicht der Industrie. In Schweiz. Vereinigung für die Förderunge des beruflichen und wissenschaftlichen Nachwuchses (Hrsg.), Talenterfassung und Nachwuchsförderung (S. 14-20). Zürich: s.n. (=Sondernummer des Mitteilungsblattes «Nachwuchsförderung - Promotion»).

Altermatt, U. (1989). Katholizismus und Moderne. Zürich: Benziger.

Arbeitsausschuss zur Förderung des wissenschaftlichen und technischen Nachwuchses (1959). Schlussbericht. Bern: s.n. (=Sondernummer des Mitteilungsblatts des Delegierten für Arbeitsbeschaffung, April 1959).

Beck, U. (1983). Jenseits von Klasse und Stand? In R. Kreckel (Hrsg.), Soziale Ungleichheiten (S. 35-74). Göttingen: Otto Schwartz (=Sonderband 2 der «Sozialen Welt»).

Beck, U. (1986). Risikogesellschaft. Frankfurt a.M.: Suhrkamp.

Becker, R. (2000). Bildungsexpansion und Bildungsbeteiligung. Zeitschrift für Erziehungswissenschaft, 3, 447-480.

Bernath, W., Löhrer, E. \& Wirthensohn, M. (1980). Die Schul- und Berufslaufbahnen 18-jähriger Zürcher Jugendlicher. Materialien IV, Band 1: Die Schul- und Berufslaufbahnen. Zürich: Erziehungsdirektion. 
Bernath, W., Wirthensohn, M. \& Löhrer, E. (1989). Jugendliche auf ihrem Weg ins Berufsleben. Bern: Haupt.

Bourdieu, P. \& Passeron, J.-C. (1971). Die Illusion der Chancengleichheit. Stuttgart: Klett.

Bourdieu, P. \& Boltanski, L. (1981). Titel und Stelle. Zum Verhältnis von Bildung und Beschäftigung. In P. Bourdieu, L. Boltanski, M. de Saint Martin \& P. Maldidier, Titel und Stelle. Über die Reproduktion der Macht (S. 89-115). Frankfurt/M.: Europäische Verlagsanstalt.

Die Studierenden an der Universität Zürich (1953). In Statistisches Büro des Kantons Zürich, Zürcher Wirtschaftsbilder, (1/2), 3-42.

Dünki, M. (1985). Akademikerbeschäftigung in den 30er-Jahren. Bern: Schweizerischer Wissenschaftsrat (=Wissenschaftspolitik, Beiheft 32).

Eidgenössisches Statistisches Amt (1938). Die Studierenden an Schweizer Hochschulen. Sondererhebung 1936. Bern: Eidgenössisches Statistisches Amt (=Beiträge zur schweizerischen Statistik, Heft 7).

Eidgenössisches Statistisches Amt (1947). Die Studierenden an Schweizer Hochschulen. Erhebung 1946. Bern: Eidgenössisches Statistisches Amt (=Beiträge zur schweizerischen Statistik, Heft 17).

Eidgenössisches Statistisches Amt (1961). Die Studierenden an Schweizer Hochschulen. Bern: Eidgenössisches Statistisches Amt (=Beiträge zur schweizerischen Statistik, Heft 31).

Eidgenössische Kommission für Nachwuchsfragen auf dem Gebiet der Geisteswissenschaften und der medizinischen Berufe sowie des Lehrerberufes auf der Mittelschule (1963). Bericht. Bern: Eidgenössisches Departement des Innern.

Erziehungsrat des Kantons Zürich (1964). Aktuelle Mittelschulfragen unter besonderer Berücksichtigung des Maturitätstypus C. Zürich: [Erziehungsdirektion].

Fend, H. (1981). Theorie der Schule. München: Urban \& Schwarzenberg.

Fend, H. (1982). Gesamtschule im Vergleich. Weinheim \& Basel: Beltz.

Frei, P. L. (1986). Chancengerechtigkeit durch öffentliche Bildung? Bern: Lang.

Gretler, A. (2000). Die Schweizerische Bildungsforschung der Nachkriegszeit. Schweizerische Zeitschrift für Bildungswissenschaften, 22, (1), 111-144.

Gurny, R., Cassée, P., Hauser, H. P. \& Meyer, A. (1984). Karrieren und Sackgassen. Chur: Rüegger.

Häberlin, U. (1972). Schweizer Gesamtschulmodelle. Basel: Beltz.

Haefeli, H., Schräder-Naef, R. \& Häfeli, K. (1979). Schulische Auslese bei Abschluss der Primarschule. Bern: Haupt.

Heid, H. (1988). Zur Paradoxie der bildungspolitischen Forderung nach Chancengleichheit. Zeitschrift für Pädagogik, 34, 1-17.

Hess, F., Latscha, F. \& Schneider, W. (1966). Die Ungleichheit der Bildungschancen. Olten: Walter.

Huber, B. (1993). Studien- und Berufswahl der Zürcher Maturandinnen und Maturanden 1992. Auswertungsbericht. Zürich: Studien- und Berufsberatung des Kantons Zürich.

Hutmacher, W. (1987). Le passeport ou la position sociale? In A. Gretler, R. Gruny, A. N. Perret-Clermont \& E. Poglia (Hrsg.), Fremde Heimat (S. 43-107). Cousset: Del Val.

Im Hof, U. (1959). Das Gymnasium und die grosse Gegenwartsaufgabe der Nachwuchsförderung in Naturwissenschaft und Technik. Gymnasium Helveticum, 14, (1), 27-41.

Kägi, E. A. (1991). Der Agglomerationsschweizer. Neue Zürcher Zeitung 22./23.6.1991.

Karrer, D., Gonon, Ph., Horvath, F. \& Rieger, A. (1991). Ende der sozialen Ungleichheit? WOZ, (23), 25-27.

Karrer, D. (2000). Die Last des Unterschieds. Wiesbaden: Westdeutscher Verlag.

Kälble, H. (1983). Soziale Mobilität und Chancengleichheit im 19. und 20. Jahrhundert. Göttingen: Vandenhoeck \& Ruprecht. 
Kronbichler, W. (1983). Die Zürcherischen Kantonsschulen 1883-1983. Zürich: Erziehungsdirektion des Kantons Zürich.

Lamprecht, M. (1991). Möglichkeiten und Grenzen schulischer Chancengleichheit in westlichen Gesellschaften. In V. Bornschier (Hrsg.), Das Ende der sozialen Schichtung? (S. 126153). Zürich: Seismo.

Lamprecht, M. \& Stamm, H. (1997). Chancengleichheit im Schweizer Bildungssystem? WIDERSPRUCH, (33), 39-44.

Lamprecht, M. \& Stamm, H. (1996). Soziale Ungleichheit im Bildungswesen. Bern: Bundesamt für Statistik

Levy, R., Joye, D., Guye, O. \& Kaufmann, V. (1997). Tous égaux? De la stratification aux représentations. Zürich: Seismo.

Liebig, B. (1997). Geschlossene Gesellschaft. Aspekte der Geschlechterungleichheit in wirtschaftlichen und politischen Fürhungsgremien der Schweiz. Chur: Rüegger.

Mantovani, L. (1994). Fremdbestimmt zur Eigenständigkeit. Chur: Rüegger.

Mayer, K. U. (1990). Lebensverläufe und sozialer Wandel. Kölner Zeitschrift für Soziologie und Sozialpsychologie Sonderheft, (31), 7-21.

Müri, F. (1971). Eltern vor der Entscheidung: Gymnasium oder Volksschule. Bern \& Frankfurt/M.: Lang.

Nabholz, W. (1981). Von Stufen und Klassen. Volksrecht 14. \& 21.10.1981.

Rektorat Universität Zürich \& Stadler, P. (1983). Die Universität Zürich 1933-1983. Zürich: NZZ-Verlag.

Rieger, A. (1992). Bildungsexpansion und soziale Chancen. Manuskript.

Schmid, Ch. (1982). Das Seminar Küsnacht. Seine Geschichte von 1832-1982. Küsnacht: Seminar Küsnacht.

Schmid-Amman, P. (1957). Die Förderung des beruflichen und akademischen Nachwuchses. Separatdruck aus Volksrecht 2., 6. und 9. Nov. 1957. Zürich: Volksrecht.

Statistische Berichte (1959). Die Schüler der öffentlichen Mittelschulen im Kanton Zürich. Zürich: Erziehungsdirektion (=Statistische Berichte des Kantons Zürich, Heft 3).

Trier, U. P. (1964). Zur Frage der Auslese für die Mittelschule. Bericht für den Erziehungsrat des Kantons Zürich. Zürich: Akademische Berufsberatung.

Ungricht, J. (1944). Zur Frage der Überfüllung der akademischen Berufe. Zürich: Zentralsekretariat Berufsberatung.

Vogt, W. (1962). Der Einfluss sozialer und wirtschaftlicher Faktoren auf die Rekrutierung der ETH-Studenten. Schweizerische Zeitschrift für Volkswirtschaft und Statistik, 98, 476-498.

Weber, K. (1985). Arbeit 2000. Bestimmungsgrössen, Wirkungszusammenhänge und Szenarien der akademischen Beschäftigung. Bern. Schweizerischer Wissenschaftsrat (=Wisschenschaftspolitik. Beiheft 32).

Wolter, St. C. (1994). Lohnt sich Bildung überhaupt? Zürich: Schweizerische Bankgesellschaft (=ECONOMIC focus, Sept. 1994). 


\section{Expansion du système de formation et disparités d'accès: l'exemple des écoles moyennes dans le canton de Zurich}

\section{Résumé}

L'analyse de l'évolution des écoles moyennes dans le canton de Zurich de 1830 à 1980 montre clairement comment les possibilités d'accès à ces formations des différents groupes sociaux se sont transformées dans le contexte d'expansion du système éducatif. Si les femmes, les populations rurales et les personnes de confession catholique présentent des situations comparables à celles des hommes, des urbains ainsi que des protestants en terme de fréquentation de ces écoles, ce n'est pas le cas des enfants de classes socio-économiques basses, de mêmes que des groupes nationaux correspondants, qui représentent le groupe le plus désavantagé sur ce plan. En arrière-fond se pose la question de savoir pourquoi la discussion sur l'égalité des chances a été évacuée quand bien même les demandes dans ce domaine n'ont pas été remplies.

\section{L'espansione della formazione e disparità di accesso: l'esempio delle scuole medie superiori del Canton Zurigo}

\section{Riassunto}

L'analisi dell'evoluzione delle scuole medio superiori del Canton Zurigo tra il 1830 e il 1980 mostra bene come le possibilità di accesso alla formazione si siano trasformate nel contesto dell'espansione della scuola: le donne, gli abitanti delle campagne e i cattolici riescono ad equiparare gli uomini, gli abitanti delle città e i protestanti. Per i figli dei ceti sociali meno abbienti - come pure per le nazionalità corrispondenti - ciò non è assolutamente il caso e infatti restano i veri svantaggiati delle scuole medio superiori zurighesi. Di fronte a questa constatazione occorre chiedersi come mai la discussione attorno al principio della parità delle possibilità si sia esaurita sebbene gli obiettivi non siano stati raggiunti.

\section{Educational expansion and inequitable participation: an example of middle schools in the Canton of Zürich}

\section{Summary}

An examination of middle schools in the Canton of Zürich between 1830 and 1980 shows clearly how, within the frame of waves of educational expansion, the participation of various social groups changed: women, rural residents, Catholics could compensate their participation in middle schools to equal that of men, urban residents and protestants. This is not the case for children of lower socioeconomic social classes - as well as coinciding nationalities. They remain the ma- 
jor disadvantaged group in the middle schools of Zürich Underlying this conclusion, the question arises as to why the discussion of equal social opportunity has been exhausted even though the demands are not fulfilled. 